\title{
Task-Related Brain Oscillation and Coherence Analysis Between High and Low Cognitive Proficiencies in Preschoolers With Attention Deficit Hyperactivity Disorder
}

\author{
I-Chun Chen \\ National Chiao Tung University \\ Chia-Ling Chen \\ Chang Gung Memorial Hospital \\ Chih-Hao Chang \\ National Chiao Tung University \\ Zuo-Cian Fan \\ National Chiao Tung University

\section{Yang Chang} \\ National Chiao Tung University \\ Dar-Shong Lin \\ Mackay Memorial Hospital \\ Cheng-Hsiu Lin \\ Doctor Lin's Psychiatric Clinic \\ Li-Wei Ko ( $\sim$ lwko@nctu.edu.tw) \\ National Chiao Tung University
}

\section{Research}

Keywords: ADHD, K-CPT, Preschoolers, Wireless EEG, Cognitive proficiency, Coherence

Posted Date: December 11th, 2020

DOI: https://doi.org/10.21203/rs.3.rs-125027/v1

License: (9) (1) This work is licensed under a Creative Commons Attribution 4.0 International License. Read Full License 


\section{Abstract}

\section{Background}

Early diagnosis and intervention is essential for children at risk of attention deficit hyperactivity disorder (ADHD). For preschool-age children with ADHD, who have heterogeneous neurobehavioral deficits, the continuous performance test and quantitative electroencephalography (EEG) can facilitate clinical diagnosis. This study investigated EEG dynamics and taskbased EEG coherence in preschoolers with ADHD, who exhibited varying cognitive proficiencies.

\section{Methods}

The participants comprised 54 preschoolers (aged 5-7 years), 18 and 16 of whom exhibited high and low cognitive proficiency (ADHD-H and ADHD-L, respectively). The remaining 20 children had typical development (TD). All the children underwent the Conners' Kiddie Continuous Performance Test and wireless EEG recording under different task conditions (rest, fast, and slow).

Results

In the slow-rate task condition, the task-related parietal delta power of preschoolers with ADHD-L was significantly higher than that of their peers with TD. In the fast-rate condition, the preschoolers with ADHD-L exhibited higher parietal delta and theta/beta ratio as well as lower parietal beta power than those with ADHD-H. Unlike those in the TD group, the alpha power values of the participants in both the ADHD groups declined from rest to the task conditions. Task- related EEG beta coherence was decreased in both ADHD groups, which were distinct with TD groups.

\section{Conclusion}

The aforementioned findings suggest that task-related brain oscillations were related to cognitive proficiency in preschool children with ADHD. The novel wireless EEG system used was demonstrated to be convenient and highly suitable for clinical use in preschool children. The EEG profiles in the present study may contain specific neural biomarkers that can assist early detection, diagnosis, and clinical planning for the treatment of ADHD in preschool children.

\section{Background}

Attention deficit hyperactivity disorder (ADHD), which is the most prevalent childhood-onset neuropsychiatric disorder, is defined as persistent inattentiveness and/or hyperactivity that impedes not only developmental but also social functioning [1-3]. Symptoms typically emerge early (at preschool age) and persist into adulthood [2-5]. ADHD diagnosis is maintained over time in most individuals diagnosed as having the condition at preschool age [6-9]. The estimated prevalence rate of preschool ADHD ranges from 2.1\% to $5.7 \%[10,11]$. In 2011, the American Academy of Pediatrics updated the ADHD clinical practice guidelines, recommending clinicians to evaluate for ADHD in all children from the ages of 4-5 years [12, 13]. Identification of preschool children at risk for ADHD and providing early intervention are imperative [5, 13-15]. The lack of clinical interviews and rating scales as well as the less structured nature of preschool settings, which is unfavorable for multi-informant assessments, make the diagnosis of preschool ADHD challenging $[16,17]$. To facilitate diagnosis, the continuous performance test (CPT) and quantitative electroencephalography (QEEG), which have the advantages of safety, low cost, high speed, and convenience, can be used to investigate individual performance on attention-demanding tasks directly [18-24].

Conners' Kiddie Continuous Performance Test (K-CPT) is a standardized task-oriented computerized modality that constitutes a useful adjunct in the early identification of ADHD and other attention-related conditions in children aged 4-7 years [25]. Significant results on at least two of the three measures of the aforementioned test and the ADHD Confidence Index provide incremental predictive validity for early diagnosis $[5,26]$. Notably, heterogeneous neurobehavioral deficits in cognitive domains have been reported in ADHD, even within a single sample [27-29]. Information on ADHD-related

Page 2/28 
neurocognitive characteristics can be determined from the response patterns obtained in the K-CPT. Moreover, K-CPT, which is superior to most commercial CPT paradigms, features varied and random time intervals between stimuli. These interstimulus intervals (ISIs), through which event rates within the same paradigm are manipulated, may pose considerable challenges to test takers. Fast-rate tasks with short ISIs require rapid information processing. By contrast, slow-rate tasks with long ISIs necessitate alertness and focus. The overall scores on the K-CPT serve as a reference for clinicians and specialists with regard to attention-related concerns and facilitate the design and planning of educational strategies and interventions [25].

QEEG, which explores electrophysiological features of brain activity, is increasingly viewed as the modality of interest through which biomarkers for the characterization of neurodevelopmental disorders can be identified [18-23, 30, 31]. Power spectral density (PSD) studies on ADHD have revealed one consistent finding: Compared with age-matched controls, participants with ADHD exhibit higher absolute and relative theta power and theta/beta ratio (TBR) in the frontocentral region of the brain at rest $[20,21,23,30,32-39]$. Regarding the other frequency power bands, despite slightly conflicting evidence, studies have reported that delta power is typically higher in individuals with ADHD than in healthy controls (HCs) [21, 31, 39-42], whereas alpha and beta power are usually lower [21, 30, 31, 40, 42]. Research has indicated that electroencephalogram (EEG) coherence, which measures similarities in electrophysiological signals between electrodes in various brain regions at specific frequency bands, may be higher or lower in individuals with ADHD than in HCs depending on variations in paired brain regions. Notably, one of the most replicated findings is that participants with ADHD aged 8-13 years exhibit higher theta coherence at rest than do HCs, predominantly in the frontal and central/parietal/occipital interhemispheric connections [21, 43].

With regard to ADHD heterogeneity, most studies have used the subtypes outlined in the Diagnostic and Statistical Manual of Mental Disorders, 4th Edition, or EEG profile clusters for grouping, computing, and comparing the band power, power ratio, or power synchronization of subpopulations of children with ADHD at rest [40,42, 44-47]. However, few studies have investigated task-based EEG coherence $[46,48,49]$ or differences in PSD between rest and a task $[50,51]$. Furthermore, to the best of our knowledge, few studies have explored the brain dynamics of subgroups of preschoolers with ADHD according to cognitive proficiency by manipulating task rate.

Most EEG studies on children with ADHD have used wet electrode systems with a stretch cap. Chu et al. [52], who used only a simple index of attention and meditation, obtained brain wave signals easily by using a commercial mobile EEG headset. In the present study, a wireless wearable EEG headset was used to quantify brain dynamics during an attention-related CPT task. The advantage of this device is that it offers more comfort than wet electrode systems do for child participants. Subsequently, we investigated EEG profiles measured using dynamic EEG during different task conditions and task-based EEG coherences among preschoolers with ADHD who had high cognitive proficiency (ADHD-H), ADHD who had low cognitive proficiency (ADHD-L), and typical development (TD). We hypothesized that EEG profiles during different task conditions would be related to ADHD heterogeneity, specifically the varying levels of cognitive proficiency. The EEG profiles in the present study may contain specific neural biomarkers that can assist early detection, diagnosis, and treatment planning for preschoolers with ADHD.

\section{Methods}

\section{Participants}

The participants comprised 54 preschoolers (aged 5-7 years), 34 of whom were diagnosed as having ADHD (26 boys and eight girls; mean age \pm standard deviation [SD]: $68.74 \pm 6.55$ months) and 20 of whom had TD (16 boys and four girls; mean age \pm SD: $67.80 \pm 5.76$ months). All the ADHD diagnoses were confirmed in the clinical setting by the senior child and adolescent psychiatrists. We recorded data on the participants' birth history, including their gestational age and birth body weight. Those who had a history of brain disorders or any other neurological disorders, chromosomal or genetic disorders, autism spectrum disorder, learning disorder, or any other mental disorder were excluded. For children with ADHD ( $n=9$; four 
children with ADHD-L and five with ADHD-H) who had been taking short-acting methylphenidates, the effects of medicine had been completely washed out before experiment initiation. The study was implemented according to policies on human research and was approved by the Research Ethics Committee of the Taiwan National Health Research Institutes (EC1070401-F). The participants' parents or guardians provided written informed consent to the academic use of the test results.

\section{Apparatus}

\section{Neuropsychological measurements}

Intelligence quotient test and behavioral rating scales. Cognitive function was evaluated using the Taiwanese version of the Wechsler Preschool and Primary Scale of Intelligence-fourth edition (WPPSI-IV) [53], which was administered by qualified psychologists. This version, which is recommended for children aged 2 years and 6 months to 7 years and 11 months, has been translated and adapted from the original WPPSI-IV [54] to account for differences in language and cultural background. We classified the participants with ADHD into groups with high and low cognitive proficiency according to their cognitive proficiency index (CPI) scores on the WPPSI-IV. Children with CPI scores of $>85$ and $\leq 85$ were classified into the ADHD-H ( $n=$ 18 ) and ADHD-L $(n=16)$ groups, respectively. Two versions of the Disruptive Behavior Disorders Rating Scale (DBDRS) for parents and teachers [55] were used to evaluate ADHD symptoms.

Conners' Kiddie continuous performance test 2nd edition. In this study, we used the 7.5-min Conners' Kiddie Continuous Performance Test 2nd Edition (K-CPT2) [25] to assess attention-related performance. The participants sat in front of a computer and were instructed to respond to targets (i.e., all objects appearing on the monitor except for soccer balls, which were nontargets) as fast and accurately as possible. As displayed in Fig. 1, the K-CPT2 involves 200 randomly presented trials divided into five blocks, each of which contains two 20-trial sub-blocks involving fast- and slow-rate tasks (1.5- and 3-s ISIs, respectively). The responses were used to compute scores reflecting various attention aspects. The nine main standardized scores generated for each participant help assessors interpret the nature of their attention problems (through different aspects). Standardized T scores were defined as having a mean of 50 with an SD of 10. Higher scores generally imply worse performance except in special cases, such as when the hit reaction time (HRT) is measured. For such assessments, d' refers to detectability or the ability to discriminate nontargets from targets. Omissions and commissions represent missed targets and incorrect responses to targets, respectively. Perseverations denote responses made within 100 ms of stimulus presentation. The HRT indicates the mean response speed of all nonperseverative responses over the test. Its variation and SD measure the consistency of the response speed. The HRT block change is the slope of the change in reaction time across the five blocks. The HRT ISI change refers to the slope of the change in reaction time between two ISIs.

\section{Wireless and wearable EEG}

The apparatus used was a novel eight-channel wearable and portable EEG system $[56,57]$ that consisted of a wireless EEG device and laptop computer (Fig. 2). The device included four components: eight semidry hygroscopic sponge-electrode sensors, an EEG data acquisition module, a Bluetooth transition module, and a rechargeable battery. The advantages of these sensors are described in the following text. First, the skin-contacting part is made of soft sponge, which prevents discomfort during EEG recording. Second, the sensors simply require a small amount of water to work, instead of the complex skin preparation and gel application process in conventional wet electrode systems. Third, the sensors can be easily and quickly assembled and fit appropriately into the original device. Fourth, the sensors provide excellent conductivity for EEG signal acquisition, with the signal quality being comparable with that of the Neuroscan system [57]. In addition, the EEG data can be used to develop a real-time detection algorithm in a more natural and comfortable state during daily life.

\section{Procedure and EEG recording}

Figure 3 illustrates the EEG recording procedure. The participants sat in a sturdy, comfortable chair in a quiet room in a relaxed state. To establish baseline resting power, resting-state EEG signals were recorded over 1 min with the participants' 
eyes open. Subsequently, an instructor informed the participants of the experimental rules and provided them the opportunity to practice prior to official initiation of the K-CPT2. Raw EEG recording data were obtained from eight electrode sites on the scalp (Fp1, Fp2, Fz, C3, C4, Pz, 01, and 02) according to the standard international 10/20 system at a 1000-Hz sampling rate. The scalp-electrode impedance of the sponge electrodes used in our previous study [57] and the present study was relatively higher (approximately up to $120 \mathrm{k} \mathrm{Ohm}$ ) than that in the conventional wet electrode system (typically approximately $20 \mathrm{k} \mathrm{Ohm}$ ). However, in a circuit design containing an amplifier with an input impedance of approximately $200 \mathrm{M} \mathrm{Ohm}$, a scalp-electrode impedance of up to $200 \mathrm{k} \mathrm{Ohm}$ is allowed for accurate signal acquisition with an approximate error rate of $0.1 \%$ [58]. In the present study, the linked earlobes were used as reference sites, and the impedance of each electrode was controlled below $100 \mathrm{k}$ Ohm throughout the EEG recording session.

\section{Data preprocessing and processing}

We used MATLAB software (MathWorks, MA, USA) to preprocess and process the EEG data. We extracted the data on rest and task-related power for analysis, eliminating artifacts from eye blinks and muscle movement. EEG experts identified and eliminated the components corresponding to muscle artifacts related to the participants' restless movements during the experiment. According the sequences of ISIs within sub-blocks (Fig. 1), we further divided the task data into two epochs: 5 min of slow-rate task data comprising all segments with a 3-s ISI and $2.5 \mathrm{~min}$ of fast-rate task data comprising all segments with a 1.5-s ISI (Fig. 4). Four frequency bands were defined for spectral analyses: delta, theta, alpha, and beta $(0-3,4-7,8-$ 12 , and $13-30 \mathrm{~Hz}$, respectively). We determined the PSD of the EEG data by using the short-time Fourier transform spectrogram function in the MATLAB signal processing toolbox. We analyzed the relative power, which was computed by dividing the absolute power in a designated frequency band by the sum of all the measured frequency bands and then multiplying the result by 100 . Relative power is independent of bone thickness, skull resistance, and impedance variability. In addition, relative power is a more discriminative variable than absolute power in ADHD evaluation studies [50, 59]. Regarding task-related coherence, the data on task-related power were Fourier-transformed using a Hamming window. We also analyzed 28 electrode pairs from eight recording sites.

\section{Statistical analysis}

The demographic and test data and the QEEG spectra of the three groups were compared using analysis of variance (ANOVA). Comparison analysis of categorical variables (e.g., sex) was conducted using chi-square tests. To avoid the type I error associated with multiple comparisons, we performed Bonferroni correction and considered differences to be significant at $p<0.01$. Raw $p$ values between 0.01 and 0.05 are presented as trend-level findings. To evaluate alterations in spectral power, we performed a three-way repeated-measures ANOVA, with condition (rest, slow-rate task, and fast-rate task) and region (Fp1, Fp2, Fz, C3, C4, Pz, 01, and 02) as within-subject factors and group (ADHD-H, ADHD-L, and TD) as a betweensubject factor. To assess between-group differences in task-related coherence, we performed nonparametric analysis with a median test. Spearman's correlation analysis was conducted to evaluate the relationship between selected task-related coherence and the DBDRS scores. The significance level was set as $p<0.05$. All the analyses were performed using IBM SPSS Statistics for Windows, version 22 (IBM Corp., Armonk, NY, USA).

\section{Results}

\section{Between-group differences in neuropsychological measurements}

Table 1 presents the comparison of the demographic and neuropsychological measurements between groups. The mean full-scale intelligence quotient of the ADHD-L group was lower than that of the TD group $(p=0.013)$; however, the verbal comprehensive index did not differ among the groups, which confirmed that all the participants completely comprehended the test instructions. CPI differed significantly between groups; specifically, the ADHD-L group performed worse than the TD and ADHD-H groups ( $p<0.001$ for both). 
Significant between-group differences were observed in the DBDRS and K-CPT2 scores. Regarding the DBDRS, the participants in the two ADHD groups were rated higher scores than those in the TD group on the inattentive dimension by both parents $(13.11 \pm 4.35$ in ADHD-H, $14.69 \pm 5.10$ in ADHD-L, $10.75 \pm 4.61$ in TD, $p=0.047)$ and teachers $(15.28 \pm 5.00$ in ADHD-H, $17.13 \pm 4.16$ in ADHD-L, $9.10 \pm 5.97$ in TD, $p<0.001)$. On the hyperactive dimension, teachers gave the participants with ADHD higher scores than those in the TD group (13.39 \pm 5.78 in ADHD-H, $14.19 \pm 6.03$ in ADHD-L, 8.25 \pm 7.74 in TD, $p=$ 0.017). Regarding the K-CPT2, the ADHD-L group exhibited inferior HRT performance (i.e., had higher HRT scores) to other groups (53.28 \pm 5.94 in ADHD-H, $63.75 \pm 7.51$ in ADHD-L, $55.05 \pm 5.84$ in TD, $p<0.001)$. The ADHD-L group performed worse (i.e., scored higher) than the TD group in detectability (54.38 \pm 8.30 in ADHD-L, $48.10 \pm 6.21$ in TD, $p=0.035$ ), omission (55.06 \pm 9.59 in ADHD-L, $47.90 \pm 9.34$ in TD, $p=0.047)$, HRT SD (55.56 \pm 10.79 in ADHD-L, $46.95 \pm 6.72$ in TD, $p=0.007)$, and HRT ISI changes (56.38 \pm 11.33 in ADHD-L, $49.00 \pm 7.12$ in TD, $p=0.031)$. Although the ADHD-H group exhibited a poorer performance than the TD group did, the K-CPT2 scores of these groups did not differ significantly (Table 1).

\section{Between-group differences in resting relative spectral power and power ratios}

Data on the relative powers of delta, theta, alpha, beta, and the TBR on eight electrodes (Fp1, Fp2, Fz, C3, C4, Pz, 01, and 02) at rest indicated no significant differences between the groups (Supplemental Table 1).

\section{Between-group differences in CPT-task-related relative spectral power and power ratios}

\section{Between-group differences in CPT-slow-rate task-related relative spectral power and power ratios}

The relative powers of delta, theta, alpha, beta, and the TBR on eight electrodes (Fp1, Fp2, Fz, C3, C4, Pz, 01, and 02) in the slow-rate task condition among the three groups were compared (Supplemental Table 2). Table 2 and Fig. 5 present the significant results from the analysis of between-group differences in the slow-rate task-related PSD. The ADHD-H group had a trend of higher delta values than the TD group did on the C4 electrode $(p=0.023)$, and the ADHD-L group had significantly higher delta values than the TD group did on the Pz electrode $(p=0.007)$. The ADHD-L group had a trend of lower alpha power values than the TD group did on the Pz electrode $(p=0.040)$. The beta power values on the Pz electrode tended to be lower in the ADHD-L group than in the TD group $(p=0.025)$. Overall, trends of between-group differences were observed in the $\operatorname{TBR}(p=0.030)$.

\section{Between-group differences in CPT-fast-rate task-related relative spectral power and power ratios}

Table 2 and Fig. 5 present the significant results from the analysis of between-group differences in the task-related PSD. The relative powers of delta, theta, alpha, beta, and the TBR on eight electrodes (Fp1, Fp2, Fz, C3, C4, Pz, 01, and 02) in the fastrate task condition among the three groups were compared (Supplemental Table 3). The delta power values were significantly higher in the ADHD-L group than in the ADHD-H group $(p=0.003)$, and presented a trend to have higher values than did the TD group on Pz electrode $(p=0.033)$. A trend which the ADHD-L group had higher values than did the TD group $(p=0.021)$ and the ADHD-H group $(p=0.023)$ on 01 electrodes was observed. Regarding the alpha power, the ADHD-L group

had a trend of lower values than did the ADHD-H group on Pz electrode $(p=0.048)$. Moreover, the beta power values on the Pz electrode were significantly lower in the ADHD-L group than in the ADHD-H group $(p=0.003)$. Significant differences between ADHD-L and ADHD-H were observed on Pz TBR $(p=0.003)$; a trend of difference between ADHD-L and ADHD-H were observed on C3 TBR $(p=0.028)$.

\section{EEG spectral power alterations of groups under three conditions}

\section{Relative delta}

Significant main effects were noted for region $(F=2.922, p=0.006)$. Specifically, a significant difference was noted between the $\mathrm{Pz}$ and 01 electrodes $(p=0.001)$. No other significant main effects or interactions were observed.

\section{Relative theta}


Significant main effects were noted for region $(F=3.019, p=0.004)$. Specifically, a significant difference was noted between the Fp2 and Pz electrodes $(p=0.042)$. Moreover, a significant interaction effect was observed between condition and region $(p=0.001)$.

\section{Relative alpha}

Significant main effects were observed for condition $(F=19.791, p<0.001)$. The post hoc test revealed significant shifts from rest to the slow-rate task and from rest to a fast-rate task ( $p<0.001$ for both). Main effects were also observed for region $(F=3.222, p=0.003)$, and the post hoc test revealed significant differences between the $\mathrm{Pz}$ and 01 electrodes $(p=$ $0.024)$ and between the $\mathrm{Pz}$ and 02 electrodes $(p=0.046)$. Significant interaction effects of condition and region were also noted $(p=0.012)$. Notably, condition and group had a significant interaction effect $(p=0.040)$, and the post hoc test indicated that the ADHD-L group experienced a decline in alpha power during the shift from rest to the slow-rate task $(p=$ $0.003)$ and from rest to a fast-rate task $(p<0.001)$. A reduction in alpha power was observed in the ADHD-H group during the transition from rest to the slow-rate task $(p<0.001)$. The aforementioned results were distinct from those obtained for the TD group, which exhibited no obvious differences in transition patterns. Figure 6 presents the transition patterns of the three groups in the three conditions.

\section{Relative beta}

Significant main effects were observed for region $(F=2.040, p=0.049)$, and a significant difference was noted between the Pz and 01 electrodes $(p=0.006)$. No other significant main effects or interactions were observed.

\section{Between-group differences in task-based coherence and their correlations with behavioral rating scores}

We compared the task-related coherence of all 28 electrode pairs in the four frequency bands among the three groups (Fig. 7). Table 3 lists the significant results from the analysis. Regarding beta coherence, the ADHD-H group had significantly lower values than the TD group on the Fp2-02 electrode pair $(p=0.028)$. On the C3-C4 electrode pair, the ADHD-L group had significantly lower beta coherence values than TD group $(p=0.022)$. The correlation analysis indicated significant negative correlations between the beta coherence on the C3-C4 electrode pair and all DBDRS scores except the inattentiveness dimension score rated by the parents (Table 4).

\section{Discussion}

To the best of our knowledge, this study is the first to construct EEG profiles under various task conditions for preschoolers with ADHD exhibiting varying cognitive proficiencies by using wireless EEG headsets. The EEG dynamics during task conditions (i.e., slow- and fast-rate tasks) were related to the cognitive proficiency of the participants with ADHD, whose taskrelated beta coherence was distinct from that of their TD peers. This result indicates that specific brain oscillations are related to the heterogeneity in the neurobehavioral functions (e.g., working memory and processing speed) of children with ADHD. The research results contribute to the understanding of task-related brain dynamics and connectivity, particularly concerning preschool ADHD. The EEG profiles in the present study may involve specific neural biomarkers that can assist early detection, diagnosis, and clinical treatment planning for preschoolers with ADHD.

Notably, differences in centroparietal delta power in the slow-rate task condition were observed between the two ADHD groups and the TD group. This result is partially inconsistent with the findings of previous studies that have indicated that the resting frontocentral theta power and TBR of children with ADHD are different from those of their TD peers [20,21, 23, 30]. This discrepancy can be attributed to between-study differences in the EEG measurement conditions. Because brain attention dysfunction is usually more evident during cognitive tasks, electrophysiological recordings conducted during task conditions can act as discriminants for the diagnosis of ADHD [60]. Attention functions involve several brain areas, and altered parietal activity in ADHD may indicate abnormalities in the posterior visual attention system that affect performance monitoring, attention reallocation, and visuospatial attention as well as motor mapping within the peripersonal space [61, 
62]. We infer that the K-CPT involves visual-input and motor response tasks that require respondents' vision attention. This phenomenon is completely different from that observed in the resting condition. Parent behavioral training (PBT) interventions have strong evidence of effectiveness in the treatment of preschoolers at risk of ADHD [4, 5, 14]. On the basis of EEG profiles, PBT should involve slow-rate task training, such as delayed conditioning practice, for children with ADHD who have various severities.

Compared with the ADHD-H group, the ADHD-L group exhibited a higher parietal delta power and TBR and lower beta power. These results can be explained by the fact that children with ADHD of low CPI are less able to process rapidly presented stimuli than those with high CPI. On the basis of the hypothesis that ADHD results from neurodevelopmental or neurocognitive deficits, various early interventions have been developed to target these presumed core deficits [5]. Specialists have employed computer-driven, play-based, and exercise programs in early intervention to enhance cognitive ability in young children with ADHD [5,63-66]. Therefore, according to these EEG profiles, in addition to behavioral training, mental processing speed (particularly on fast-rate tasks) tailored to the individual should constitute a crucial component of cognitive training for young children with ADHD who have low CPI scores [67].

In this study, both ADHD groups exhibited an overall reduction in alpha powers in the shift from rest to the slow-rate task. Moreover, in the shift from rest to the fast-rate task, the ADHD-L group exhibited a reduction in alpha power. These results are in line with those of a study by Swartwood et al. [51], in which the alpha power in posterior regions at baseline was higher in boys (aged 9-11 years) with ADHD than in age-matched controls. The aforementioned researchers also reported reductions in alpha power in the left frontal region in the ADHD group during the cognitive task of reading. Our findings suggest that preschoolers with ADHD exhibiting both high and low CPI have difficulty maintaining alertness and performing stable executive function during shifts to slow-rate task conditions. Furthermore, the levels of cortical activation in children with ADHD of low $\mathrm{CPI}$ are insufficient to meet the cognitive requirements during shifts from rest to fast-rate task conditions. The EEG transition profiles indicate that PBT should be performed through approaches such as practice repetition, strategy guidance, and task prompting to enhance the ability to adjust to slow-rate task shifting for all children with ADHD, regardless of cognitive proficiency, to adjust to slow-rate task shifting. PBT is also recommended for parents of children with ADHD of low CPI to help the children improve their ability to shift to fast-rate task conditions.

Intrahemispheric fronto-occipital and interhemispheric central beta coherence were lower in the ADHD groups than in the TD group. Furthermore, significant correlations were observed between DBDRS scores and interhemispheric central beta coherence. Most EEG coherence studies on ADHD have examined resting states [43, 45, 68]. Few studies have assessed taskbased connectivity $[46,48,69]$. EEG coherence in children with ADHD may be increased or decreased depending on the paired brain regions and conditions for recording [21]. Our findings are partially compatible with those of previous studies that observed reduced interhemispheric temporal alpha coherence and central/parietal/occipital beta coherence $[43,45,68]$. This partial discrepancy may be due to between-study differences in the methodology, the sample, and task characteristics. Because beta coherence is a robust and specific marker of motor status [70], reduced task-related beta coherence may be indicative of impaired motor responses in children with ADHD, especially those with low CPI. Accordingly, EEG coherence may constitute another neural marker for early detection of ADHD in preschoolers.

The wearable wireless semidry-electrode EEG recording system used in the present study has none of the drawbacks of conventional wet electrode EEG systems. Specialists typically find it challenging to monitor the attention status of young children during task performance due to poor compliance and unnatural situations. In our experiences with using the semidry-electrode system, children do not need to endure the lengthy preparation procedure involved in wet electrode systems (which exacerbates impatient and fidgety behavior in children). Notably, the participants noted that wearing the system was comfortable, similar to wearing stylish headphones. They also mentioned that the wireless design made task performance feel easy and free; thus, the adopted system promoted the cooperation of the participants in the measurements. Most importantly, the data collected using the adopted system is equivalent in quality to that produced by wet electrode systems. In summary, the adopted system is highly suitable for clinical use in young children. 
The relatively small sample size used is a limitation of this study. Nevertheless, we believe that our findings are clinically relevant to the understanding of the underlying mechanisms of ADHD, particularly preschool ADHD, and can serve as a reference for clinicians and researchers with regard to the potential neural biomarkers.

\section{Conclusion}

The EEG profiles in the present study reflect the heterogeneity of ADHD. EEG dynamics and coherences are related to cognitive heterogeneity in individuals with ADHD. EEG dynamics in the slow-rate task and in the shift from rest to the slowrate task were impaired in children with either high and low CPI scores. EEG dynamics in the fast-rate task and in the shift from rest to the fast-rate task were impaired in children with ADHD who had low CPI scores. The acquisition of EEG profiles by using the novel wireless system is convenient and efficient for clinical use in children. These profiles can provide clinicians and researchers with information on potential neural markers that aid in the early detection, diagnosis, and planning of interventions for preschool ADHD, including individual training and educational programs. Future studies should attempt to use the aforementioned system to measure brain activity and provide real-time neurofeedback during game therapy according to the selected neural biomarkers. This system can also be applied to various attention-related tasks in which children engage, such as reading and writing.

\section{Declarations}

\section{Ethics approval and consent to participate}

The study was implemented according to policies on human research and was approved by the Research Ethics Committee of the Taiwan National Health Research Institutes (EC1070401-F). The participants' parents or guardians provided written informed consent to the academic use of the test results.

\section{Consent for publication}

Not applicable.

\section{Availability of data and materials}

The datasets used and/or analysed during the current study are available from the corresponding author on reasonable request.

\section{Competing interests}

The authors declare that they have no competing interests.

\section{Funding}

This work was supported in part by the Aiming for the Top University Plan of National Chiao Tung University, the Ministry of Education, Taiwan, under Contracts 109W211, MMH-CT-10809, and MMH-CT-10903.

\section{Authors' contributions}

ICC participated in the conception, study design, experimental setup, data collection, data analysis, data interpretation, and draft of this manuscript. CLC participated in the conception, data interpretation and revising of this manuscript. CHC participated in the signal processing, data analysis, and data interpretation. ZCF participated in the signal processing and data analysis. YC participated in the study design and experimental setup. DSL participated in the conception. CHL participated in the data collection. LWK participated in the conception, study design, data interpretation, and the revising of this manuscript. All authors read and approved the final manuscript. 


\section{Acknowledgements}

This manuscript was edited by Wallace Academic Editing.

\section{Author details}

${ }^{1}$ International Ph. D. Program in Interdisciplinary Neuroscience, College of Biological Science and Technology, National Chiao Tung University, No.1001, Daxue Rd., East Dist., Hsinchu City 300093, Taiwan. ${ }^{2}$ Department of Physical Medicine and Rehabilitation, Ton-Yen General Hospital, No.69, Xianzheng 2nd Rd., Zhubei City, Hsinchu County 302048, Taiwan. ${ }^{3}$ Department of Physical Medicine and Rehabilitation, Chang Gung Memorial Hospital, Linkou, No.5, Fuxing St., Guishan Dist., Taoyuan City 333423, Taiwan. ${ }^{4}$ Graduate Institute of Early Intervention, College of Medicine, Chang Gung University, No.259, Wenhua 1st Rd., Guishan Dist., Taoyuan City 333323, Taiwan. ${ }^{5}$ Department of Biological Science and Technology, National Chiao Tung University, No.1001, Daxue Rd., East Dist., Hsinchu City 300093, Taiwan. ${ }^{6}$ Institute of Bioinformatics and Systems Biology, National Chiao Tung University, No.1001, Daxue Rd., East Dist., Hsinchu City 300093, Taiwan. ${ }^{7}$ Center for Intelligent Drug Systems and Smart Bio-devices (IDS2B), National Chiao Tung University, No.1001, Daxue Rd., East Dist., Hsinchu City 300093, Taiwan. ${ }^{8}$ Department of Pediatrics, Mackay Memorial Hospital, No.92, Sec. 2, Zhongshan N. Rd., Zhongshan Dist., Taipei City 104217, Taiwan. ${ }^{9}$ Department of Medicine, Mackay Medical College, No.46, Sec. 3, Zhongzheng Rd., Sanzhi Dist., New Taipei City 252005, Taiwan. ${ }^{10}$ Doctor Lin's Psychiatric Clinic, No.38, Xinguang Rd., East Dist., Hsinchu City 300041, Taiwan. ${ }^{11}$ Drug Development and Value Creation Research Center, Kaohsiung Medical University, No.100, Shiquan 1st Rd., Sanmin Dist., Kaohsiung City 807378, Taiwan.

\section{References}

1. Feldman HM, Reiff MI. Clinical practice. Attention deficit-hyperactivity disorder in children and adolescents. N Engl J Med. 2014;370(9):838-46.

2. Association AP. Diagnostic and statistical manual of mental disorders (DSM-5®). Washington, DC: American Psychiatric Association; 2013.

3. Thapar A, Cooper M. Attention deficit hyperactivity disorder. Lancet. 2016;387(10024):1240-50.

4. Tandon M, Pergjika A. Attention deficit hyperactivity disorder in preschool-age children. Child Adolesc Psychiatr Clin N Am. 2017;26(3):523-38.

5. Halperin JM, Marks DJ. Practitioner review: assessment and treatment of preschool children with attentiondeficit/hyperactivity disorder. J Child Psychol Psychiatry. 2019;60:930-43.

6. Bufferd SJ, Dougherty LR, Carlson GA, Rose S, Kleinet DN. Psychiatric disorders in preschoolers: continuity from ages 3 to 6. Am J Psychiatry 2012;169(11):1157-64.

7. Bunte TL, Schoemaker K, Hessen DJ, van der Heijden PGM, Matthys W. Stability and change of ODD, CD and ADHD diagnosis in referred preschool children. J Abnorm Child Psychol. 2014;42(7):1213-24.

8. Law EC, Sideridis GD, Prock LA, Sheridan MA. Attention-deficit/hyperactivity disorder in young children: predictors of diagnostic stability. Pediatrics. 2014;133(4):659-67.

9. Lahey BB, Lee SS, Sibley MH, Applegate B, Molina BSG, Pelham WE. Predictors of adolescent outcomes among 4-6-yearold children with attention-deficit/hyperactivity disorder. J Abnorm Psychol. 2016;125(2):168-81.

10. Danielson ML, Bitsko RH, Ghandour RM, Holbrook JM, Kogan MD, Blumberg SJ. Prevalence of parent-reported ADHD diagnosis and associated treatment among US children and adolescents, 2016. J Clin Child Adolesc Psychol 2018;47(2):199-212.

11. Lavigne JV, Lebailly SA, Hopkins J, Gouze KR, Binns HJ. The prevalence of ADHD, ODD, depression, and anxiety in a community sample of 4-year-olds. J Clin Child Adolesc Psychol. 2009;38(3):315-28. 
12. Wolraich M, Hagan JF, Allan C, Chan E, Davison D, Earls M, et al. ADHD: clinical practice guideline for the diagnosis, evaluation, and treatment of attention-deficit/hyperactivity disorder in children and adolescents. Pediatrics. 2011;128(5):1007-22.

13. Fiks AG, Ross ME, Mayne SL, Song L, Liu W, Steffes J, et al. Preschool ADHD diagnosis and stimulant use before and after the 2011 AAP practice guideline. Pediatrics. 2016;138(6):e20162025.

14. Charach A, Carson P, Fox S, Ali MU, Beckett J, Lim CG. Interventions for preschool children at high risk for ADHD: a comparative effectiveness review. Pediatrics. 2013;131(5):e1584-604.

15. Takayanagi N, Yoshida S, Yasuda S, Adachi M, Kaneda-Osato A, Tanaka M, et al. Psychometric properties of the Japanese ADHD-RS in preschool children. Res Dev Disabil. 2016;55:268-78.

16. Merkt J, Siniatchkin M, Petermann F. Neuropsychological measures in the diagnosis of ADHD in preschool: can developmental research inform diagnostic practice? J Atten Disord. 2020;24(11):1588-604.

17. O'Neill S, Schneiderman RL, Rajendran K, Marks DJ, Halperin JM. Reliable ratings or reading tea leaves: can parent, teacher, and clinician behavioral ratings of preschoolers predict ADHD at age six? J Abnorm Child Psychol. 2014;42(4):623-34.

18. Jeste SS, Frohlich J, Loo SK. Electrophysiological biomarkers of diagnosis and outcome in neurodevelopmental disorders. Curr Opin Neurol. 2015;28(2):110-16.

19. Kim J, Lee Y, Han D, Min K, Kim D, Lee C. The utility of quantitative electroencephalography and integrated visual and auditory continuous performance test as auxiliary tools for the attention deficit hyperactivity disorder diagnosis. Clin Neurophysiol. 2015;126(3):532-40.

20. Lenartowicz A, Loo SK. Use of EEG to diagnose ADHD. Curr Psychiatry Rep. 2014;16(11):498.

21. Alba G, Pereda E, Manas S, Mendez LD, Gonzalez A, Gonzalez JJ. Electroencephalography signatures of attentiondeficit/hyperactivity disorder: clinical utility. Neuropsychiatr Dis Treat. 2015;11:2755-69.

22. Olbrich S, van Dinteren R, Arns M. Personalized medicine: review and perspectives of promising baseline EEG biomarkers in major depressive disorder and attention deficit hyperactivity disorder. Neuropsychobiology. 2015;72(34):229-40.

23. McVoy M, Lytle S, Fulchiero E, Aebi ME, Adeleye O, Sajatovic M. A systematic review of quantitative EEG as a possible biomarker in child psychiatric disorders. Psychiatry Res. 2019;279:331-44.

24. Hall CL, Valentine AZ, Groom MJ, Walker GM, Sayal K, Daley D, et al. The clinical utility of the continuous performance test and objective measures of activity for diagnosing and monitoring ADHD in children: a systematic review. Eur Child Adolesc Psychiatry. 2016;25(7):677-99.

25. Conners CNT. Conners' Kiddie continuous performance test 2nd Edition. New York: Multi-Health Systems Inc; 2015.

26. Breaux RP, Griffith SF, Harvey EA. Preschool neuropsychological measures as predictors of later attention deficit hyperactivity disorder. J Abnorm Child Psychol. 2016;44(8):1455-71.

27. Coghill DR, Seth S, Matthews K. A comprehensive assessment of memory, delay aversion, timing, inhibition, decision making and variability in attention deficit hyperactivity disorder: advancing beyond the three-pathway models. Psychol Med. 2014;44(9):1989-2001.

28. Luo Y, Weibman D, Halperin JM, Li X. A review of heterogeneity in Attention Deficit/Hyperactivity Disorder (ADHD). Front Hum Neurosci. 2019;13:42.

29. Sjöwall D, Thorell LB. A critical appraisal of the role of neuropsychological deficits in preschool ADHD. Child Neuropsychol. 2019;25(1):60-80.

30. Loo SK, Makeig S. Clinical utility of EEG in attention-deficit/hyperactivity disorder: a research update. Neurotherapeutics. 2012;9(3):569-87.

31. Newson JJ, Thiagarajan TC. EEG frequency bands in psychiatric disorders: a review of resting state studies. Front Hum Neurosci. 2018;12:521. 
32. Shi T, Li X, Song J, Zhao N, Sun C, Xia W, et al. EEG characteristics and visual cognitive function of children with attention deficit hyperactivity disorder (ADHD). Brain Dev. 2012;34(10):806-11.

33. Buyck I, Wiersema JR. Resting electroencephalogram in attention deficit hyperactivity disorder: developmental course and diagnostic value. Psychiatry Res. 2014;216(3):391-7.

34. Snyder SM, Hall JR. A meta-analysis of quantitative EEG power associated with attention-deficit hyperactivity disorder. J Clin Neurophysiol. 2006;23(5):440-55.

35. Snyder SM, Quintana H, Sexson SB, Knott P, Haque AFM, Reynolds DA. Blinded, multi-center validation of EEG and rating scales in identifying ADHD within a clinical sample. Psychiatry Res. 2008;159(3):346-58.

36. Snyder SM, Rugino TA, Hornig M, Stein MA. Integration of an EEG biomarker with a clinician's ADHD evaluation. Brain Behav. 2015;5(4):e00330.

37. Lansbergen MM, Arns NM, van Dongen-Boomsma M, Spronk D, Buitelaar JK. The increase in theta/beta ratio on restingstate EEG in boys with attention-deficit/hyperactivity disorder is mediated by slow alpha peak frequency. Prog Neuropsychopharmacol Biol Psychiatry. 2011;35(1):47-52.

38. Clarke AR, Barry RJ, Johnstone S. Resting state EEG power research in attention-deficit/hyperactivity disorder: a review update. Clin Neurophysiol. 2020;131(7):1463-79.

39. Markovska-Simoska S, Pop-Jordanova N. Quantitative EEG in children and adults with attention deficit hyperactivity disorder: comparison of absolute and relative power spectra and theta/beta ratio. Clin EEG Neurosci. 2017;48(1):20-32.

40. Clarke AR, Barry RJ, McCarthy R, Selikowitz M. Age and sex effects in the EEG: differences in two subtypes of attentiondeficit/hyperactivity disorder. Clin Neurophysiol. 2001;112(5):815-26.

41. Kim JW, Lee J, Kim BN, Kang T, Min KJ, Han DH, et al. Theta-phase gamma-amplitude coupling as a neurophysiological marker of attention deficit/hyperactivity disorder in children. Neurosci Lett. 2015;603:25-30.

42. Clarke AR, Barry RJ, McCarthy R, Selikowitz M. EEG-defined subtypes of children with attention-deficit/hyperactivity disorder. Clin. Neurophysiol. 2001;112(11):2098-105.

43. Barry RJ, Clarke AR, Hajos M, Dupuy FE, McCarthy R, Selikowitz M. EEG coherence and symptom profiles of children with attention-deficit/hyperactivity disorder. Clin Neurophysiol. 2011;122(7):1327-32.

44. Clarke AR, Barry RJ, Dupuy FE, Heckel LD, McCarthy R, Selikowitz M, et al. Behavioural differences between EEG-defined subgroups of children with attention-deficit/hyperactivity disorder. Clin. Neurophysiol. 2011;122(7):1333-41.

45. Robbie JC, Clarke AR, Barry RJ, Dupuy FE, McCarthy R, Selikowitz M. Coherence in children with AD/HD and excess alpha power in their EEG. Clin Neurophysiol. 2016;127(5):2161-6.

46. Mazaheri A, Fassbender C, Coffey-Corina S, Hartanto TA, Schweitzer JB, Mangun GR. Differential oscillatory electroencephalogram between attention-deficit/hyperactivity disorder subtypes and typically developing adolescents. Biol Psychiatry. 2014;76(5):422-9.

47. Ogrim G, Kropotov J, Hestad K. The quantitative EEG theta/beta ratio in attention deficit/hyperactivity disorder and normal controls: sensitivity, specificity, and behavioral correlates. Psychiatry Res. 2012;198(3):482-8.

48. Silberstein RB, Pipingas A, Farrow M, Levy F, Stough CK, Camfield DA. Brain functional connectivity abnormalities in attention-deficit hyperactivity disorder. Brain Behav. 2016;6(12):e00583.

49. Michelini G, Jurgiel J, Bakolis I, Cheung CHM, Asherson P, Loo SK, et al. Atypical functional connectivity in adolescents and adults with persistent and remitted ADHD during a cognitive control task. Transl Psychiatry. 2019;9(1):137.

50. Nazari MA, Wallois F, Aarabi A, Berquin P. Dynamic changes in quantitative electroencephalogram during continuous performance test in children with attention-deficit/hyperactivity disorder. Int J Psychophysiol. 2011;81(3):230-6.

51. Swartwood JN, Swartwood MO, Lubar JF, Timmermann DL. EEG differences in ADHD-combined type during baseline and cognitive tasks. Pediatr Neurol. 2003;28(3):199-204.

52. Chu KC, Lu HK, Huang MC, Lin SJ, Liu WI, Huang YS, et al. Using mobile electroencephalography and actigraphy to diagnose attention-deficit/hyperactivity disorder: case-control comparison study. JMIR Ment Health. 2020;7(6):e12158.

Page $12 / 28$ 
53. Chen J, Chen HT. Manual for the Wechsler preschool and primary scale of intelligence-IV. Taiwan: Chinese Behavioral Science Corporation; 2013.

54. Wechsler D. WPPSI-IV: wechsler preschool and primary scale of Intelligence - fourth edition: manual. San Antonio, CA: Pearson Psychological Corporation; 2014.

55. Barkley RA, Murphy KR. Attention-deficit hyperactivity disorder: a clinical workbook. New York, NY: Guilford Publications; 2005.

56. Ko L, Chang Y, Wu PL, Lu YC, Yeh CL, Chen YJ. Novel moisture retention sponge electrodes for developing a wireless EEG SSVEP-based BCI system. 2018 International Automatic Control Conference (CACS); 2018.

57. Ko LW, Chang Y, Wu PL, Tzou HA, Chen SF, Tang SC, et al. Development of a smart helmet for strategical BCI applications. Sensors (Basel). 2019;19(8):1867.

58. Ferree TC, Luu P, Russell GS, Tucker DM. Scalp electrode impedance, infection risk, and EEG data quality. Clin Neurophysiol. 2001;112(3):536-44.

59. Barry RJ, Clarke AR, Johnstone SJ. A review of electrophysiology in attention-deficit/hyperactivity disorder: I. qualitative and quantitative electroencephalography. Clin Neurophysiol. 2003;114(2):171-83.

60. Sohn H, Kim I, Lee W, Peterson BS, Hong H, Chae JH, et al. Linear and non-linear EEG analysis of adolescents with attention-deficit/hyperactivity disorder during a cognitive task. Clin Neurophysiol. 2010;121(11):1863-70.

61. Pliszka SR, McCracken JT, Maas JW. Catecholamines in attention-deficit hyperactivity disorder: current perspectives. J Am Acad Child Adolesc Psychiatry. 1996;35(3):264-72.

62. Cherkasova MV, Hechtman L. Neuroimaging in attention-deficit hyperactivity disorder: beyond the frontostriatal circuitry. Can J Psychiatry. 2009;54(10):651-64.

63. Tamm L, Nakonezny PA, Hughes CW. An open trial of a metacognitive executive function training for young children with ADHD. J Atten Disord. 2014;18(6):551-9.

64. Re AM, Capodieci A, Cornoldi C. Effect of training focused on executive functions (attention, inhibition, and working memory) in preschoolers exhibiting ADHD symptoms. Front Psychol. 2015;6:1161.

65. Capodieci A, Gola ML, Cornoldi C, Re AM. Effects of a working memory training program in preschoolers with symptoms of attention-deficit/hyperactivity disorder. J Clin Exp Neuropsychol. 2018;40(1):17-29.

66. Maeir A, Fisher O, Bar-llan RT, Boas N, Berger I, Landau YE. Effectiveness of Cognitive-Functional (Cog-Fun) occupational therapy intervention for young children with attention deficit hyperactivity disorder: a controlled study. Am J Occup Ther. 2014;68(3):260-7.

67. Aubrey HF, Ronald AK. Therapist's guide to learning and attention disorders. practical resources for the mental health professional. Amsterdam: Academic Press; 2003.

68. Barry RJ, Clarke AR, McCarthy R, Selikowitz M. EEG coherence in attention-deficit/hyperactivity disorder: a comparative study of two DSM-IV types. Clin Neurophysiol. 2002;113(4):579-85.

69. Murias M, Swanson JM, Srinivasan R. Functional connectivity of frontal cortex in healthy and ADHD children reflected in EEG coherence. Cereb Cortex. 2007;17(8):1788-99.

70. Wu J, Quinlan EB, Dodakian L, McKenzie A, Kathuria N, Zhou RJ, et al. Connectivity measures are robust biomarkers of cortical function and plasticity after stroke. Brain. 2015;138(8):2359-69.

\section{Tables}

\section{Table 1 Demographic characteristics and K-CPT values of the participants}




\begin{tabular}{|c|c|c|c|c|c|c|c|}
\hline Mean $\pm S . D$. & $\begin{array}{l}\text { ADHD-H }(n= \\
18)\end{array}$ & ADHD-L $(n=16)$ & $\operatorname{TD}(n=20)$ & $p$ & $\begin{array}{l}\text { ADHD- } \\
\text { H vs } \\
\text { TD } \\
p\end{array}$ & $\begin{array}{l}\text { ADHD-L } \\
\text { vs TD } \\
p\end{array}$ & $\begin{array}{l}\text { ADHD-H } \\
\text { vs } \\
\text { ADHD-L } \\
p\end{array}$ \\
\hline Age & $68.50 \pm 6.14$ & $69.06 \pm 7.18$ & $67.80 \pm 5.76$ & 0.836 & & & \\
\hline $\begin{array}{l}\text { Sex (male: } \\
\text { female) }\end{array}$ & $12: 6$ & $14: 2$ & $16: 4$ & 0.330 & & & \\
\hline GA & $38.83 \pm 0.99$ & $38.63 \pm 1.63$ & $38.85 \pm 1.14$ & 0.846 & & & \\
\hline BBW & $3243.89 \pm 366.89$ & $3106.75 \pm 465.14$ & $3100.70 \pm 454.62$ & 0.532 & & & \\
\hline FIQ & $99.61 \pm 12.79$ & $89.13 \pm 14.27$ & $103.28 \pm 14.08$ & $0.013^{*}$ & 1.000 & $0.013^{*}$ & 0.092 \\
\hline $\mathrm{VCl}$ & $101.39 \pm 12.36$ & $96.94 \pm 14.70$ & $103.89 \pm 14.64$ & 0.349 & & & \\
\hline CPI & $99.21 \pm 6.93$ & $75.80 \pm 7.29$ & $101.75 \pm 13.47$ & $<0.001^{* *}$ & 1.000 & $<0.001 * *$ & $<0.001 * *$ \\
\hline DBRSiP & $13.75 \pm 4.12$ & $14.69 \pm 5.10$ & $10.75 \pm 4.61$ & $0.034^{*}$ & 0.059 & $0.014^{*}$ & 0.569 \\
\hline DBRShP & $14.06 \pm 5.43$ & $12.69 \pm 6.82$ & $9.55 \pm 5.58$ & 0.073 & $0.028 *$ & 0.122 & 0.516 \\
\hline DBRSiT & $15.28 \pm 5.00$ & $17.13 \pm 4.16$ & $9.10 \pm 5.97$ & $<0.001^{* \star}$ & $0.001 * \star$ & $<0.001 *$ & 0.303 \\
\hline DBRShT & $13.39 \pm 5.78$ & $14.19 \pm 6.03$ & $8.25 \pm 7.74$ & $0.017 *$ & $0.021 *$ & $0.010 *$ & 0.728 \\
\hline$d^{\prime}$ & $50.39 \pm 7.02$ & $54.38 \pm 8.30$ & $48.10 \pm 6.21$ & $0.039 *$ & 0.986 & $0.035^{*}$ & 0.332 \\
\hline omission & $49.44 \pm 6.29$ & $55.06 \pm 9.59$ & $47.90 \pm 9.34$ & $0.043^{*}$ & 1.000 & $0.047 *$ & 0.182 \\
\hline commission & $48.56 \pm 7.86$ & $52.00 \pm 10.00$ & $46.85 \pm 7.85$ & 0.203 & & & \\
\hline perseveration & $49.22 \pm 5.80$ & $50.25 \pm 7.77$ & $46.10 \pm 3.74$ & 0.090 & & & \\
\hline HRT & $53.28 \pm 5.94$ & $63.75 \pm 7.51$ & $55.05 \pm 5.84$ & $<0.001^{* *}$ & 1.000 & $0.001^{* \star}$ & $<0.001 * *$ \\
\hline HRT SD & $50.50 \pm 6.36$ & $55.56 \pm 10.79$ & $46.95 \pm 6.72$ & $0.009 * *$ & 0.539 & $0.007 * \star$ & 0.217 \\
\hline variability & $52.11 \pm 10.68$ & $51.75 \pm 9.03$ & $48.10 \pm 7.01$ & 0.321 & & & \\
\hline $\begin{array}{l}\text { HRT block } \\
\text { change }\end{array}$ & $50.00 \pm 6.51$ & $50.63 \pm 11.38$ & $49.85 \pm 4.64$ & 0.953 & & & \\
\hline $\begin{array}{l}\text { HRT ISI } \\
\text { change }\end{array}$ & $52.06 \pm 5.86$ & $56.38 \pm 11.33$ & $49.00 \pm 7.12$ & $0.036 *$ & 0.778 & $0.031^{*}$ & 0.401 \\
\hline
\end{tabular}

${ }^{* *} p<0.01,{ }^{*} p<0.05$

K-CPT, Conners' Kiddie Continuous Performance Test; SD, standard deviation; ADHD, attention deficit hyperactivity disorder; ADHD-H, ADHD with high cognitive proficiency; ADHD-L, ADHD with low cognitive proficiency ; TD, typical development; GA, gestational age; BBW, birth body weight; FSIQ, full-scale intelligence quotient; $\mathrm{VCl}$, verbal comprehension index; $\mathrm{CPI}$, cognitive proficiency index; DBDRS-P-i, Disruptive Behavior Disorders Rating Scale parent version inattentiveness dimension; DBDRSP-h, Disruptive Behavior Disorders Rating Scale parent version hyperactivity dimension; DBDRS-T-i, Disruptive Behavior Disorders Rating Scale teacher version inattentiveness dimension; DBDRS-T-h, Disruptive Behavior Disorders Rating Scale teacher version hyperactivity dimension; d', detectability; HRT, hit reaction time; ISI, interstimulus interval

Table 2Between-group differences in the PSD under different frequency and conditions 


\begin{tabular}{|c|c|c|c|c|c|c|c|}
\hline Mean $\pm S . D$ & $\begin{array}{l}\text { ADHD-H }(n= \\
18)\end{array}$ & $\begin{array}{l}\text { ADHD-L }(n= \\
16)\end{array}$ & $\begin{array}{l}\text { TD } \\
20)\end{array}(n=$ & $\begin{array}{l}\text { Overall } \\
p\end{array}$ & $\begin{array}{l}\text { ADHD-H vs } \\
\text { TD } \\
p\end{array}$ & $\begin{array}{l}\text { ADHD-L vs } \\
\text { TD } \\
p\end{array}$ & $\begin{array}{l}\text { ADHD-H vs } \\
\text { ADHD-L } \\
p\end{array}$ \\
\hline \multicolumn{8}{|l|}{$\begin{array}{l}\text { Slow rate } \\
\text { task }\end{array}$} \\
\hline C4 delta & $33.54 \pm 1.46$ & $33.01 \pm 0.96$ & $\begin{array}{l}32.27 \\
\pm 1.65\end{array}$ & $0.026 *$ & $0.023^{*}$ & 0.375 & 0.822 \\
\hline Pz delta & $33.20 \pm 0.89$ & $33.95 \pm 1.38$ & $\begin{array}{l}32.60 \\
\pm 1.41\end{array}$ & $0.009 * *$ & 0.427 & $0.007^{* *}$ & 0.265 \\
\hline Pz alpha & $22.62 \pm 0.45$ & $22.29 \pm 0.47$ & $\begin{array}{l}22.72 \\
\pm 0.57\end{array}$ & $0.039 *$ & 1.000 & $0.040 *$ & 0.189 \\
\hline Pz beta & $16.25 \pm 1.34$ & $15.34 \pm 1.60$ & $\begin{array}{l}16.85 \\
\pm 1.89\end{array}$ & $0.030 *$ & 0.810 & $0.025^{*}$ & 0.335 \\
\hline Pz TBR & $1.73 \pm 0.19$ & $1.87 \pm 0.23$ & $\begin{array}{l}1.68 \\
\pm 0.23\end{array}$ & $0.030 *$ & 1.000 & $0.029 *$ & 0.198 \\
\hline \multicolumn{8}{|l|}{$\begin{array}{l}\text { Fast rate } \\
\text { task }\end{array}$} \\
\hline Pz delta & $32.69 \pm 1.47$ & $34.23 \pm 1.00$ & $\begin{array}{l}33.11 \\
\pm 1.26\end{array}$ & $0.003^{* \star}$ & 0.926 & $0.033^{*}$ & $0.003^{* *}$ \\
\hline Pz alpha & $22.79 \pm 0.81$ & $22.26 \pm 0.41$ & $\begin{array}{l}22.62 \\
\pm 0.55\end{array}$ & $0.049 *$ & 1.000 & 0.266 & $0.048 *$ \\
\hline Pz beta & $16.65 \pm 1.92$ & $14.88 \pm 0.67$ & $\begin{array}{l}16.06 \\
\pm 1.52\end{array}$ & $0.004^{\star *}$ & 0.678 & 0.067 & $0.003^{\star *}$ \\
\hline 01 delta & $32.26 \pm 1.29$ & $33.40 \pm 1.15$ & $\begin{array}{l}32.27 \\
\pm 1.12\end{array}$ & $0.010 *$ & 1.000 & $0.021 *$ & $0.023^{*}$ \\
\hline 01 beta & $17.01 \pm 1.88$ & $15.67 \pm 1.18$ & $\begin{array}{l}16.82 \\
\pm 1.70\end{array}$ & $0.045^{\star}$ & 1.000 & 0.123 & 0.062 \\
\hline C3 TBR & $1.64 \pm 0.24$ & $1.84 \pm 0.18$ & $\begin{array}{l}1.73 \\
\pm 0.19\end{array}$ & $0.033^{*}$ & 0.611 & 0.398 & $0.028 *$ \\
\hline Pz TBR & $1.70 \pm 0.23$ & $1.93 \pm 0.12$ & $\begin{array}{l}1.77 \\
\pm 0.20\end{array}$ & $0.004^{* *}$ & 0.696 & 0.064 & $0.003^{* *}$ \\
\hline
\end{tabular}

${ }^{* *} p<0.01$ is the significance level; ${ }^{*} p<0.05$ is the trend level, Significant differences are indicated in bold. P $<0.05 / 5, P S D$, power spectral density, SD, standard deviation; ADHD, attention deficit hyperactivity disorder; ADHD-H, ADHD with high cognitive proficiency; ADHD-L, ADHD with low cognitive proficiency; TD, typical development; TBR, theta/beta ratio

Table 3 Between-group differences in task-related coherence in various frequency bands on different paired electrodes 


\begin{tabular}{|c|c|c|c|c|c|c|c|}
\hline Median & $\begin{array}{l}\text { ADHD-H }(n= \\
18)\end{array}$ & $\begin{array}{l}\text { ADHD-L }(n= \\
16)\end{array}$ & $\operatorname{TD}(n=20)$ & $\begin{array}{l}\text { Overall } \\
p\end{array}$ & $\begin{array}{l}\text { ADHD-H vs } \\
\text { TD } \\
p\end{array}$ & $\begin{array}{l}\text { ADHD-L vs } \\
\text { TD } \\
p\end{array}$ & $\begin{array}{l}\text { ADHD-H vs } \\
\text { ADHD-L } \\
p\end{array}$ \\
\hline \multirow{2}{*}{$\begin{array}{l}\text { C3-01 } \\
\text { delta }\end{array}$} & 0.11 & 0.18 & 0.18 & $0.050 *$ & 0.581 & 1.000 & 0.118 \\
\hline & $(0.011-0.475)$ & $(0.006-0.924)$ & $\begin{array}{l}(0.005- \\
0.974)\end{array}$ & & & & \\
\hline \multirow{2}{*}{$\begin{array}{l}\text { Fp2-Fz } \\
\text { alpha }\end{array}$} & 0.35 & 0.20 & 0.51 & $0.049 *$ & 0.581 & 0.133 & 0.508 \\
\hline & $(0.003-0.902)$ & $(0.001-0.838)$ & $\begin{array}{l}(0.003- \\
0.601)\end{array}$ & & & & \\
\hline \multirow{2}{*}{$\begin{array}{l}\text { Fp2-02 } \\
\text { beta }\end{array}$} & 0.06 & 0.08 & 0.19 & $0.034^{*}$ & $0.028 *$ & 0.133 & 1.000 \\
\hline & $\triangle 0.003-0.944 \rrbracket$ & $\begin{array}{l}₫ 0.009- \\
0.707 \rrbracket\end{array}$ & $\begin{array}{l}₫ 0.002- \\
0.915 \rrbracket\end{array}$ & & & & \\
\hline \multirow[t]{2}{*}{$\mathrm{Fz}-\mathrm{O} 2$ beta } & 0.12 & 0.04 & 0.29 & $0.049 *$ & 0.581 & 0.133 & 1.000 \\
\hline & $\nabla 0.007-0.871 \rrbracket$ & $(0.002-0.862)$ & $\begin{array}{l}(0.002- \\
0.979)\end{array}$ & & & & \\
\hline \multirow{2}{*}{$\begin{array}{l}\text { C3-C4 } \\
\text { beta }\end{array}$} & 0.38 & 0.36 & 0.53 & $0.010^{*}$ & 0.581 & $0.022 *$ & 1.000 \\
\hline & $\llbracket 0.063-0.689 \rrbracket$ & $\begin{array}{l}\otimes 0.187- \\
0.932 \rrbracket\end{array}$ & $\begin{array}{l}\otimes 0.012- \\
0.997 \rrbracket\end{array}$ & & & & \\
\hline
\end{tabular}

${ }^{*} p<0.05$, Significant differences are indicated in bold.

ADHD, attention deficit hyperactivity disorder; ADHD-H, ADHD with high cognitive proficiency; ADHD-L, ADHD with low cognitive proficiency

Table 4 Spearman's correlations between specific coherence and behavioral rating scales $(n=54)$

\begin{tabular}{|llll|}
\hline & \multicolumn{3}{l}{ Beta coherence } \\
\cline { 2 - 4 } & Fp2-02 & Fz-02 & C3-C4 \\
\hline DBRSiP & 0.049 & -0.039 & -0.167 \\
\hline DBRShP & 0.122 & 0.014 & $-\mathbf{0 . 3 4 0 *}$ \\
\hline DBRSiT & 0.037 & 0.029 & $-\mathbf{0 . 2 9 8 *}$ \\
\hline DBRShT & 0.112 & 0.007 & $-\mathbf{0 . 4 0 1 * *}$ \\
\hline
\end{tabular}

Significant correlations are indicated in bold

${ }^{* *} p<0.01 ;{ }^{*} p<0.05$

Abbreviations: DBDRS-P-i, Disruptive Behavior Disorders Rating Scale parent version inattentiveness dimension; DBDRS-P-h, Disruptive Behavior Disorders Rating Scale parent version hyperactivity dimension; DBDRS-T-i, Disruptive Behavior Disorders Rating Scale teacher version inattentiveness dimension; DBDRS-T-h, Disruptive Behavior Disorders Rating Scale teacher version hyperactivity dimension 
Data representation: correlation coefficient of task-related beta coherence on selected paired electrodes (horizontal axis) and four behavioral rating scores (vertical axis) of all the participants

\section{Figures}
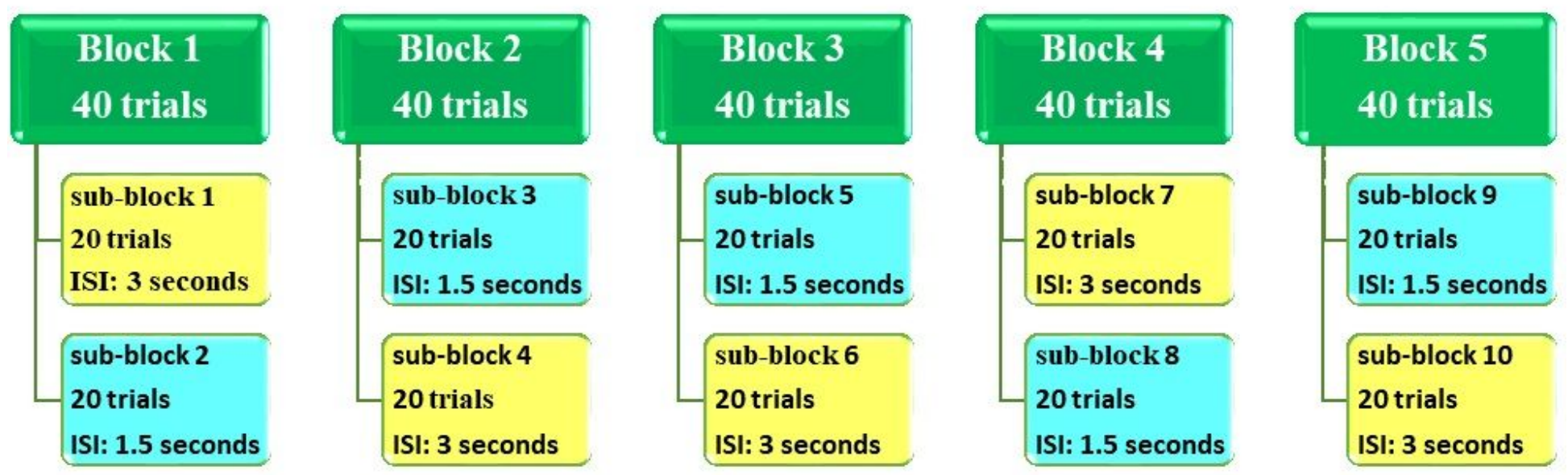

Figure 1

Structure of the K-CPT2. The K-CPT2 involves 200 randomly presented trials divided into five blocks, each of which contains two 20-trial sub-blocks containing fast- and slow-rate tasks (1.5-s and 3-s ISIs), respectively [25]. K-CPT2, Conners' Kiddie Continuous Performance Test 2nd Edition 

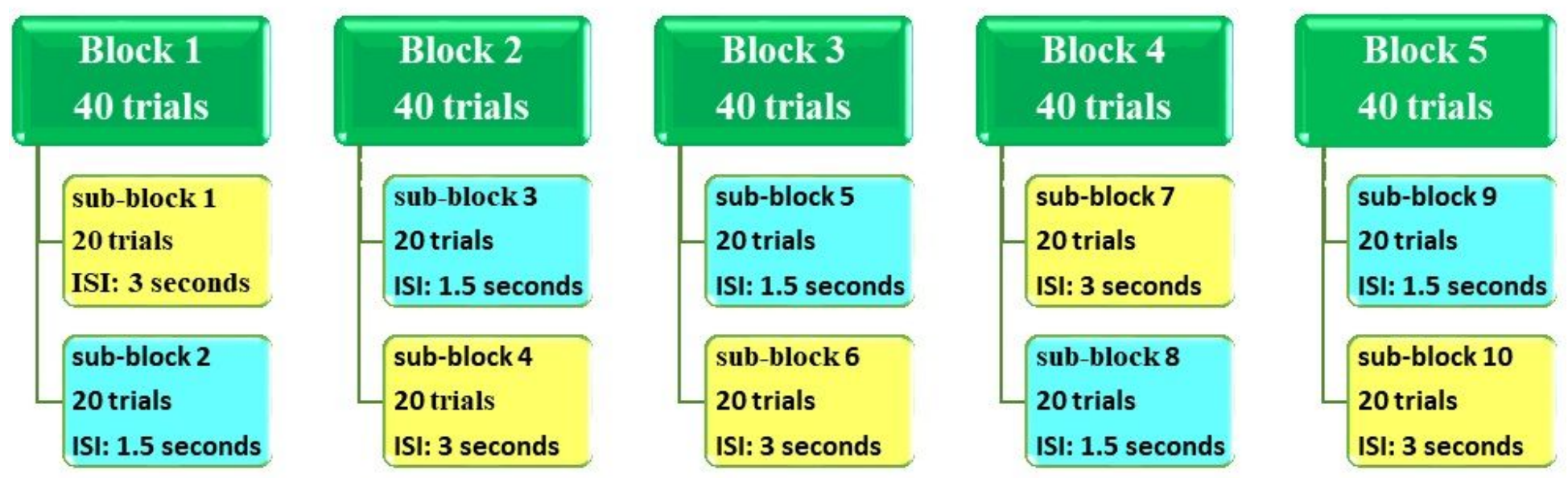

Figure 1

Structure of the K-CPT2. The K-CPT2 involves 200 randomly presented trials divided into five blocks, each of which contains two 20-trial sub-blocks containing fast- and slow-rate tasks (1.5-s and 3-s ISIs), respectively [25]. K-CPT2, Conners' Kiddie Continuous Performance Test 2nd Edition 


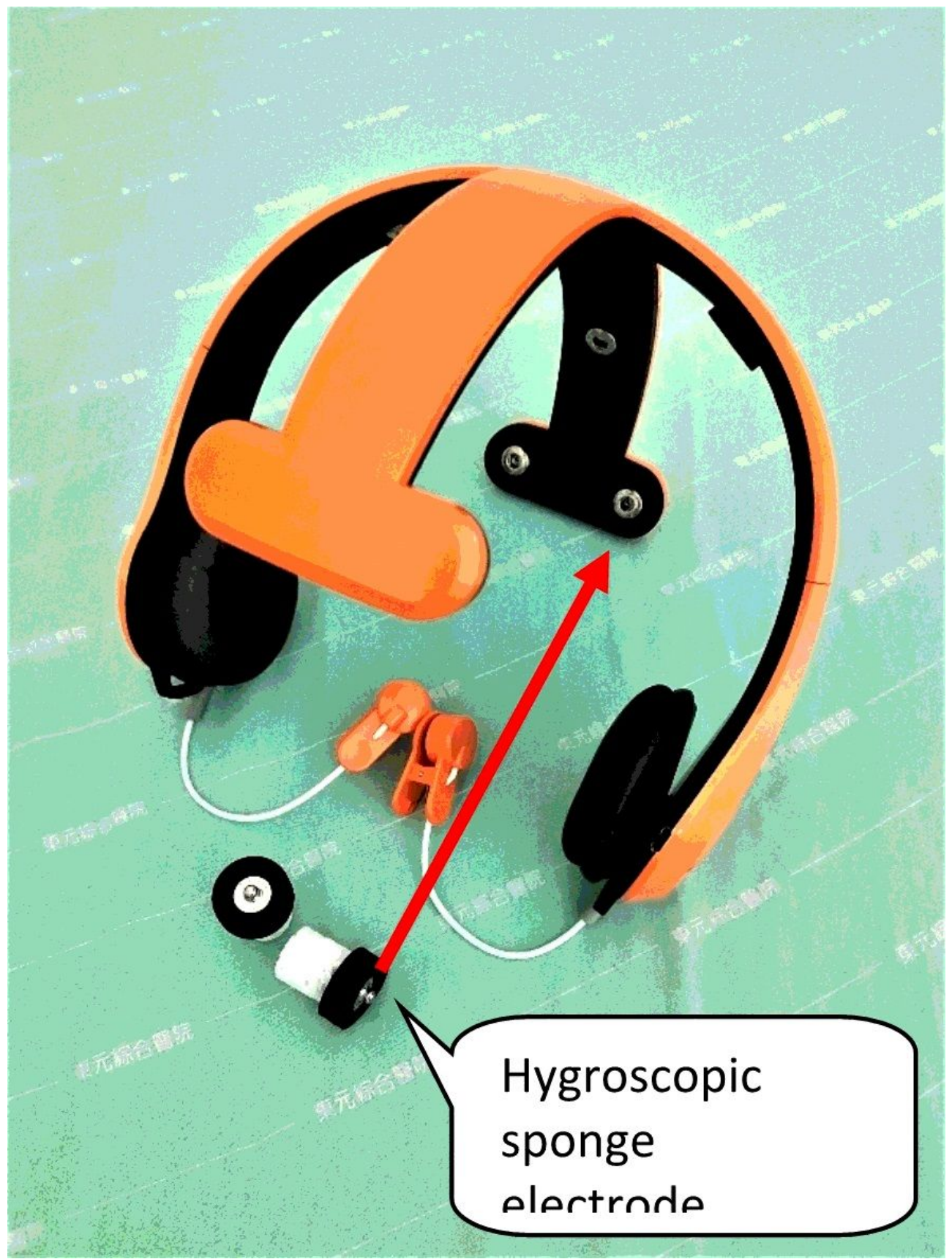

Figure 2

Wireless EEG device. A wireless and wearable EEG headset (top) and novel hygroscopic sponge electrodes (bottom). EEG, electroencephalogram 


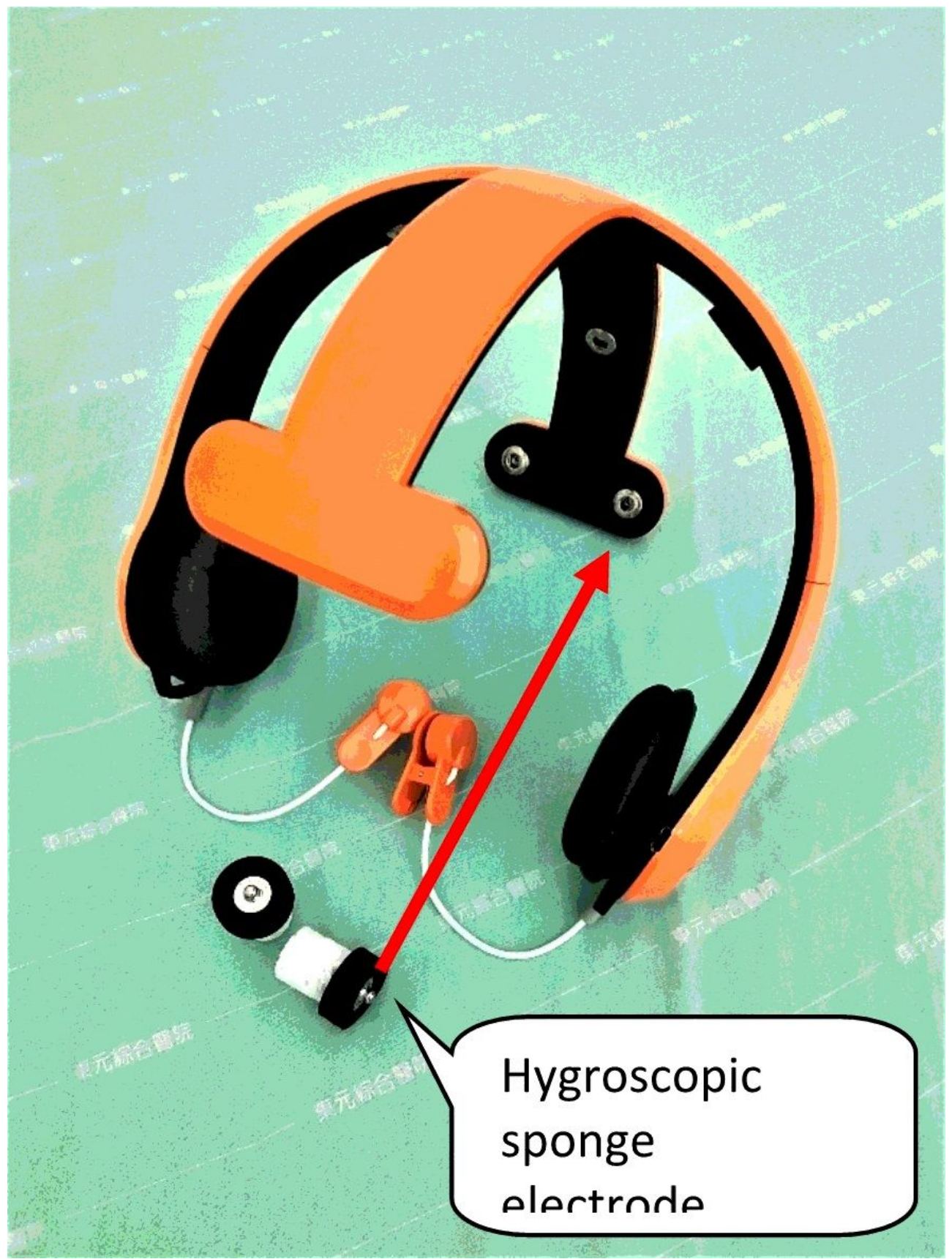

Figure 2

Wireless EEG device. A wireless and wearable EEG headset (top) and novel hygroscopic sponge electrodes (bottom). EEG, electroencephalogram 

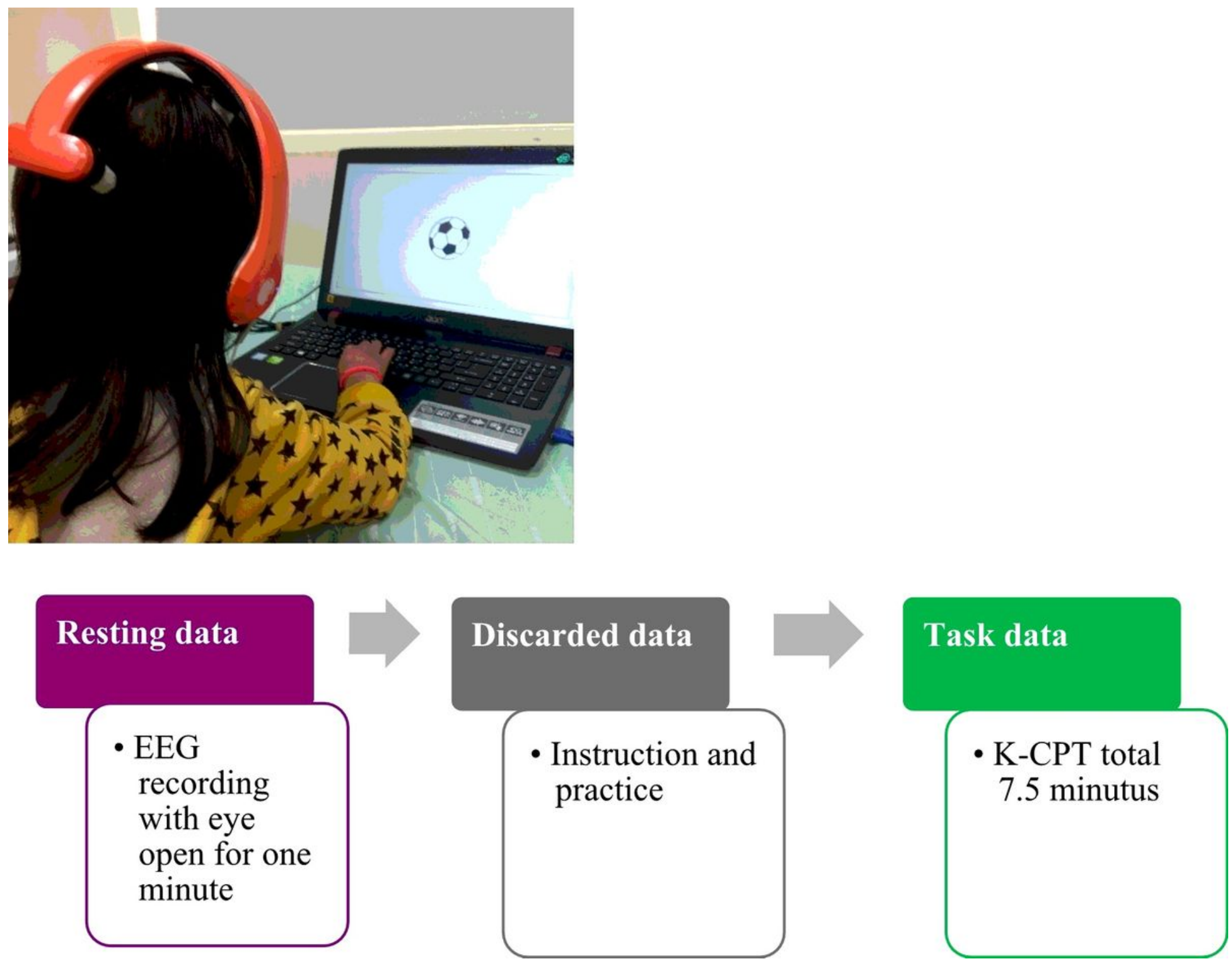

\section{Figure 3}

EEG recording procedure. A participant wearing the wireless EEG device sat in front of a computer. She was instructed to respond as fast and as accurately as possible when any pictures, except soccer balls, appeared on the monitor (left). Two EEG epochs were recorded during the assessment. The first epoch was $1 \mathrm{~min}$ in a resting state, and the second epoch was the 7.5-min K-CPT2 (right). EEG, electroencephalogram; K-CPT2, Conners' Kiddie Continuous Performance Test Second Edition 

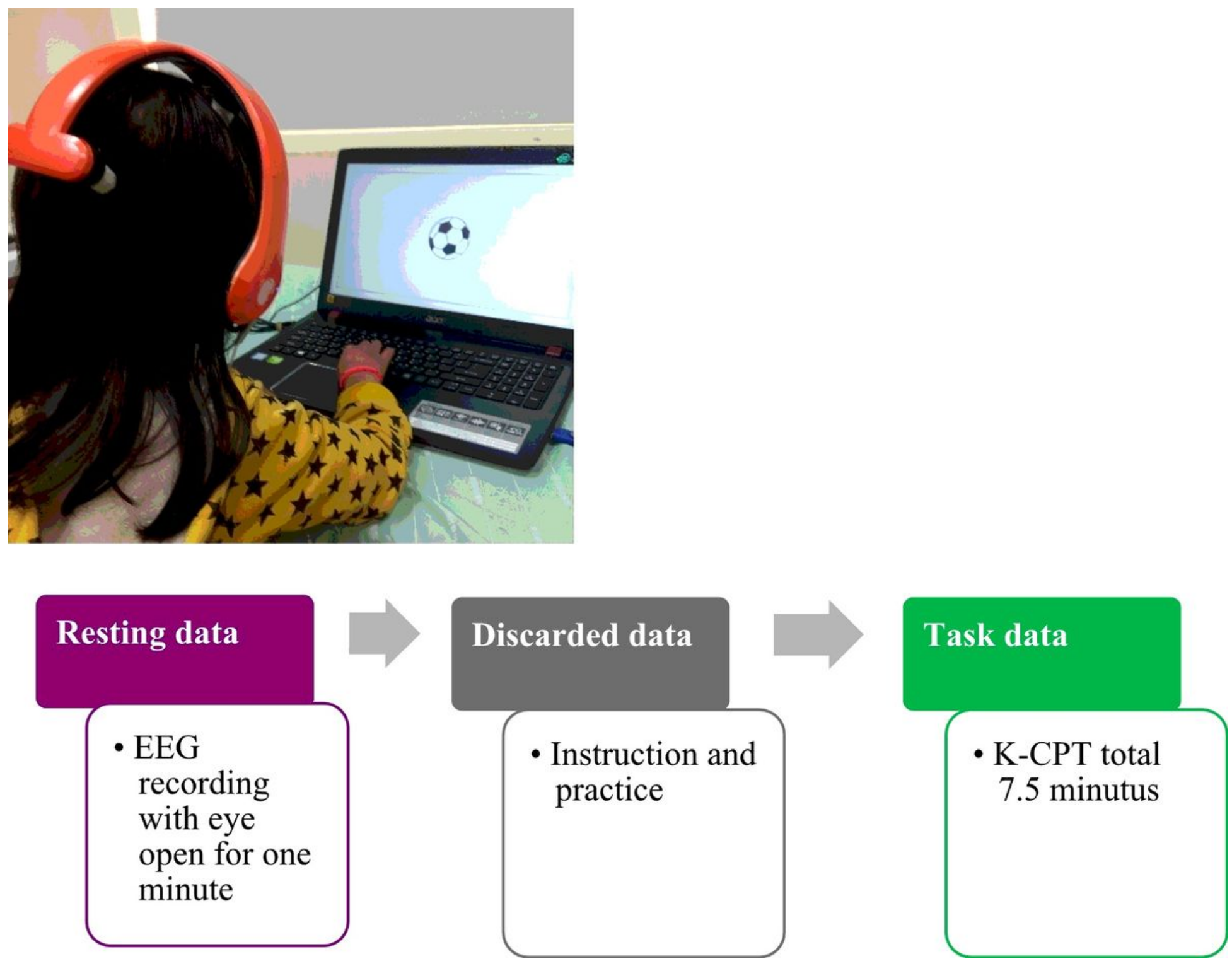

\section{Figure 3}

EEG recording procedure. A participant wearing the wireless EEG device sat in front of a computer. She was instructed to respond as fast and as accurately as possible when any pictures, except soccer balls, appeared on the monitor (left). Two EEG epochs were recorded during the assessment. The first epoch was $1 \mathrm{~min}$ in a resting state, and the second epoch was the 7.5-min K-CPT2 (right). EEG, electroencephalogram; K-CPT2, Conners' Kiddie Continuous Performance Test Second Edition 


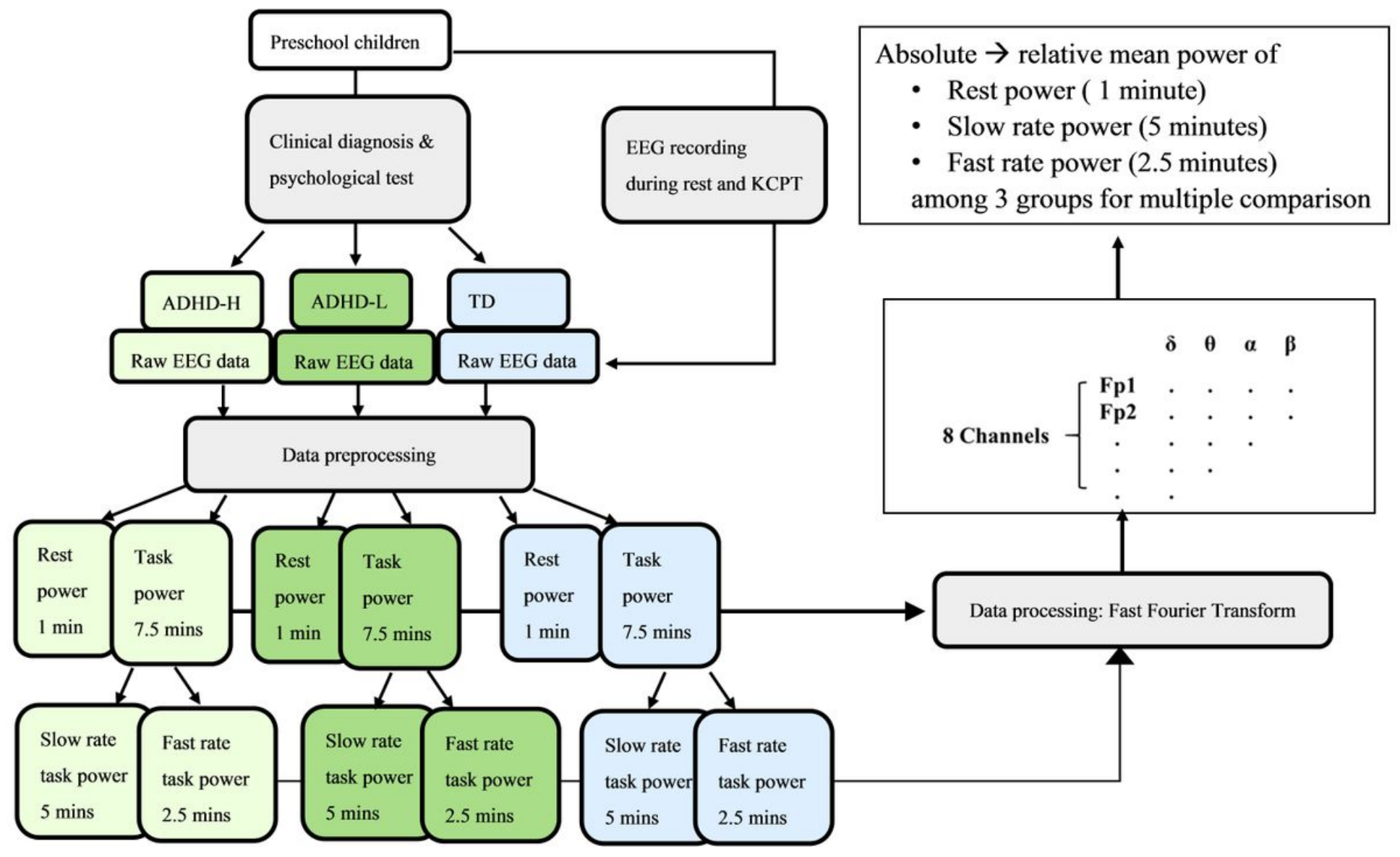

Figure 4

Data preprocessing and processing. K-CPT, Conners' Kiddie Continuous Performance Test; ADHD, attention deficit hyperactivity disorder; ADHD-H, ADHD with high cognitive proficiency; ADHD-L, ADHD with low cognitive proficiency; TD, typical development; EEG, electroencephalogram 


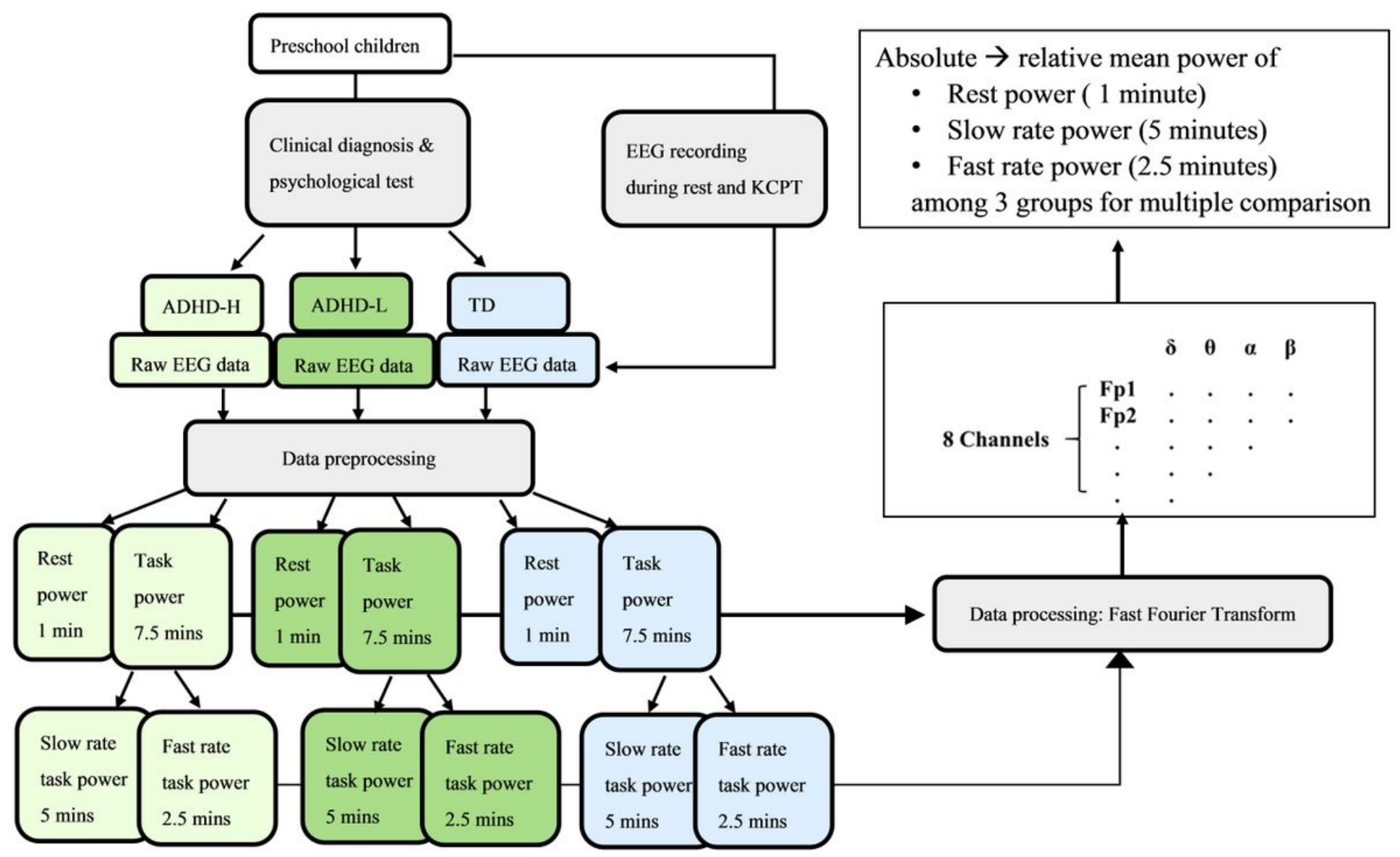

Figure 4

Data preprocessing and processing. K-CPT, Conners' Kiddie Continuous Performance Test; ADHD, attention deficit hyperactivity disorder; ADHD-H, ADHD with high cognitive proficiency; ADHD-L, ADHD with low cognitive proficiency; TD, typical development; EEG, electroencephalogram

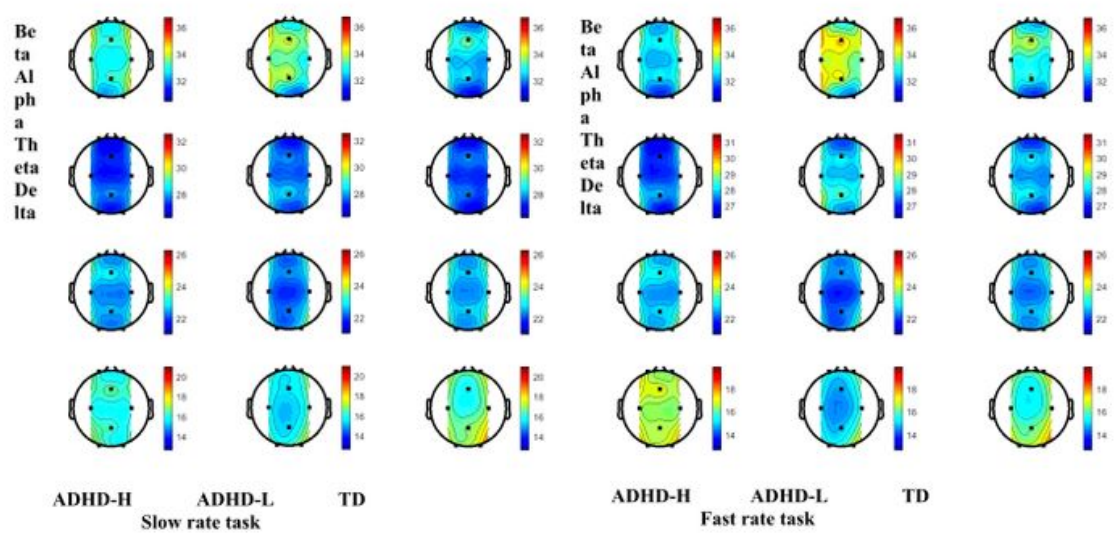

Figure 5

Topography of relative power in two conditions: slow-rate task (left) and fast-rate task (right). ADHD: ADHD: attention deficit hyperactivity disorder; ADHD-H: ADHD with high cognitive proficiency; ADHD-L: ADHD with low cognitive proficiency; TD: typical development 


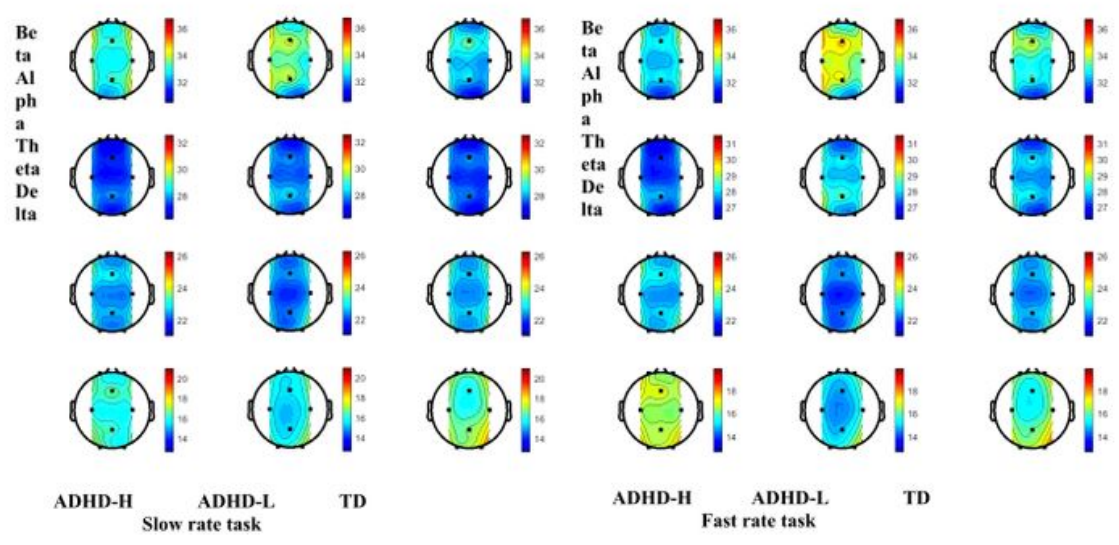

Figure 5

Topography of relative power in two conditions: slow-rate task (left) and fast-rate task (right). ADHD: ADHD: attention deficit hyperactivity disorder; ADHD-H: ADHD with high cognitive proficiency; ADHD-L: ADHD with low cognitive proficiency; TD: typical development

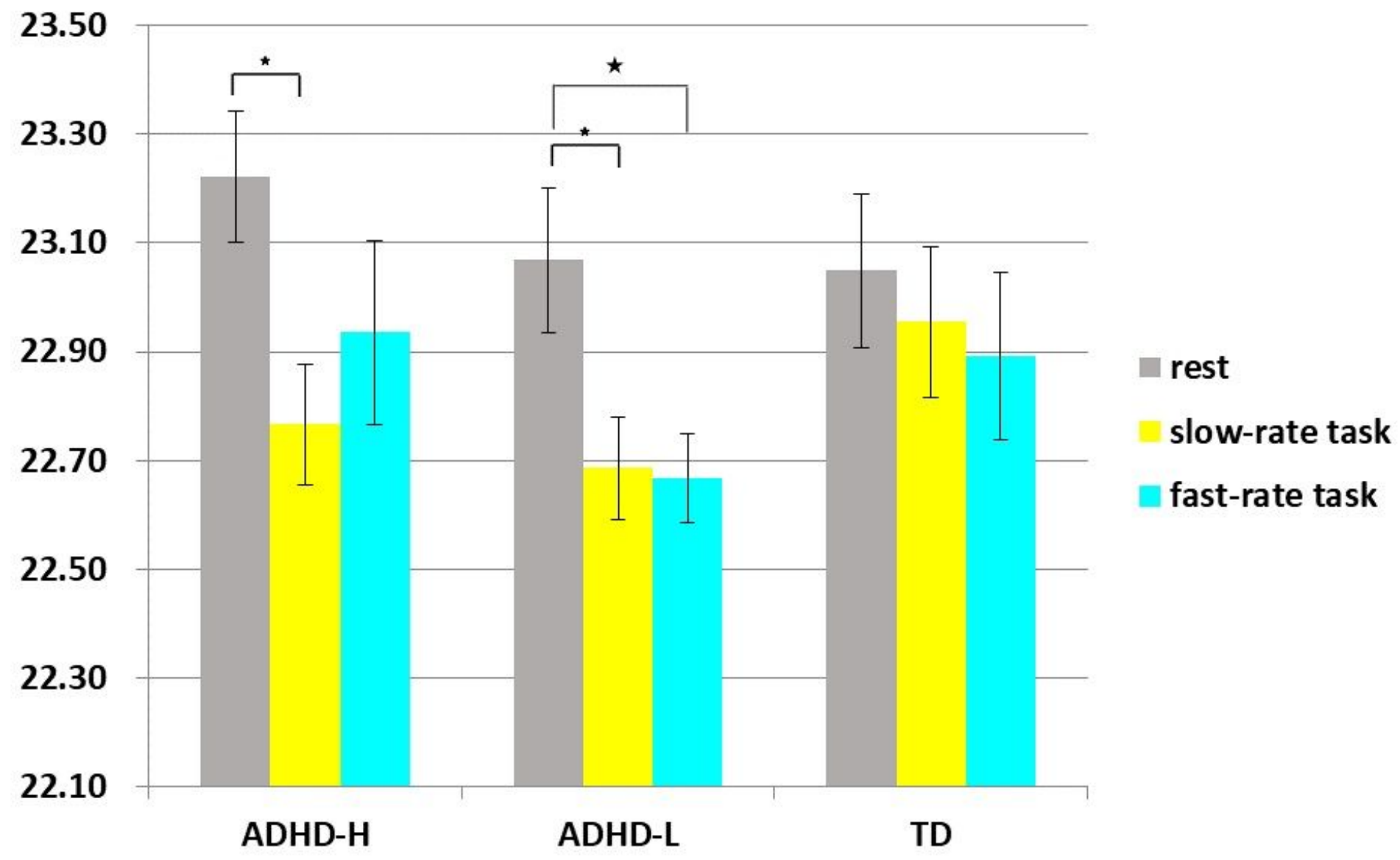

Figure 6

Relative alpha power alterations among groups under three conditions (rest, slow-rate, and fast-rate task). ADHD, attention deficit hyperactivity disorder; ADHD-H, ADHD with high cognitive proficiency; ADHD-L, ADHD with low cognitive proficiency; TD, typical development. * $\mathrm{p}<0.01$ 


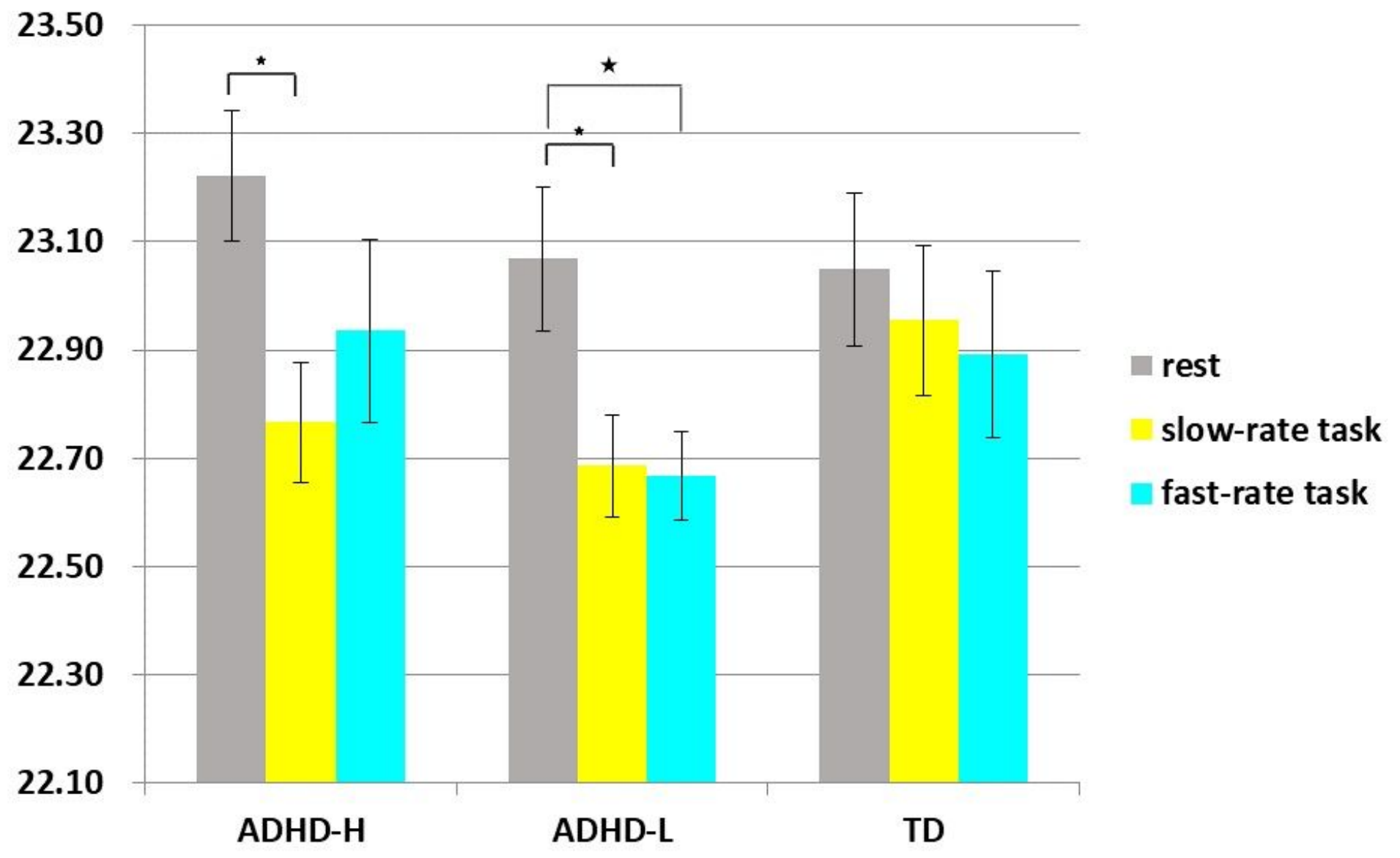

Figure 6

Relative alpha power alterations among groups under three conditions (rest, slow-rate, and fast-rate task). ADHD, attention deficit hyperactivity disorder; ADHD-H, ADHD with high cognitive proficiency; ADHD-L, ADHD with low cognitive proficiency; TD, typical development. * $p<0.01$ 

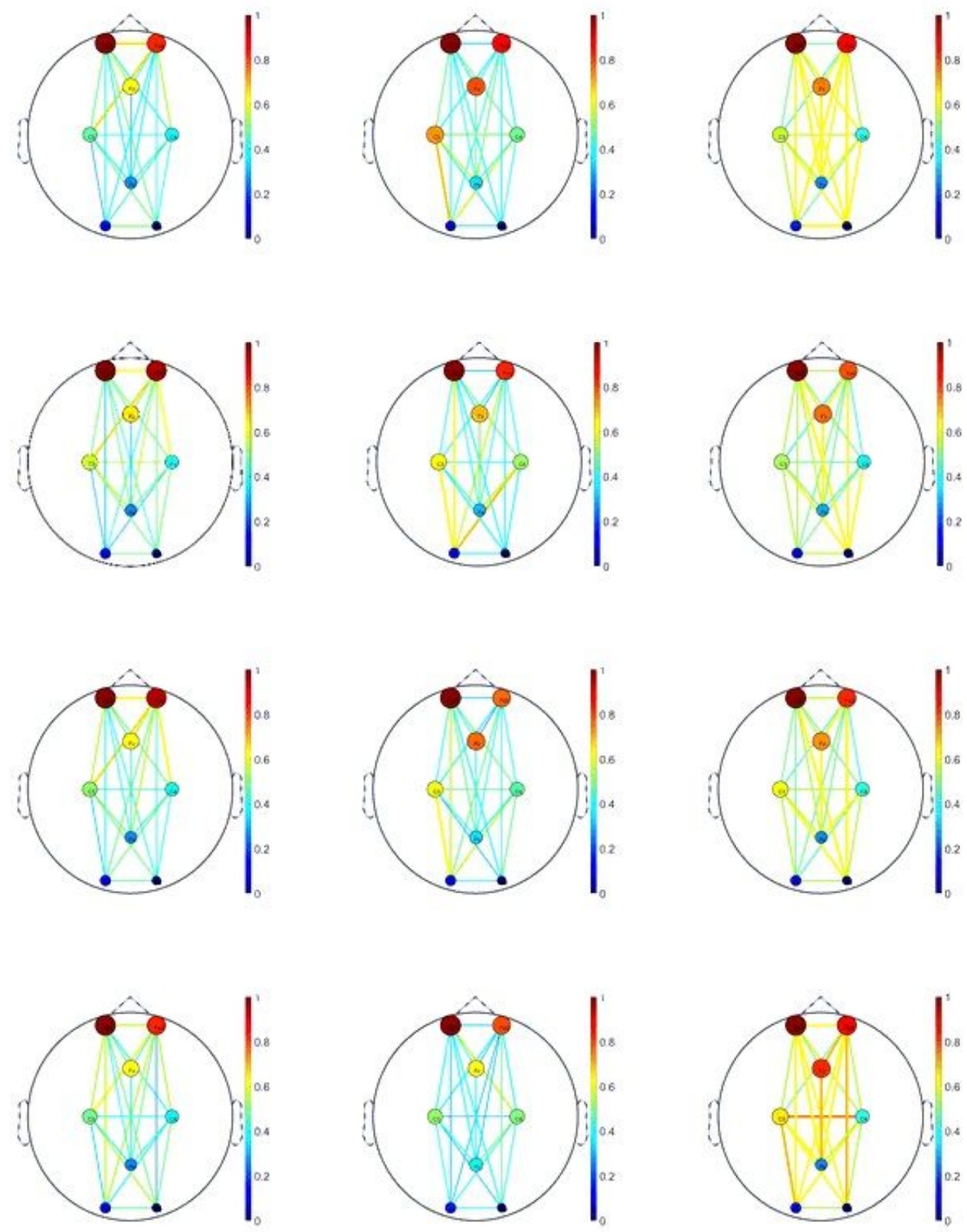

Figure 7

Task-related coherence in the three groups. ADHD, ADHD: attention deficit hyperactivity disorder; ADHD-H, ADHD with high cognitive proficiency; ADHD-L, ADHD with low cognitive proficiency; TD, typical development 

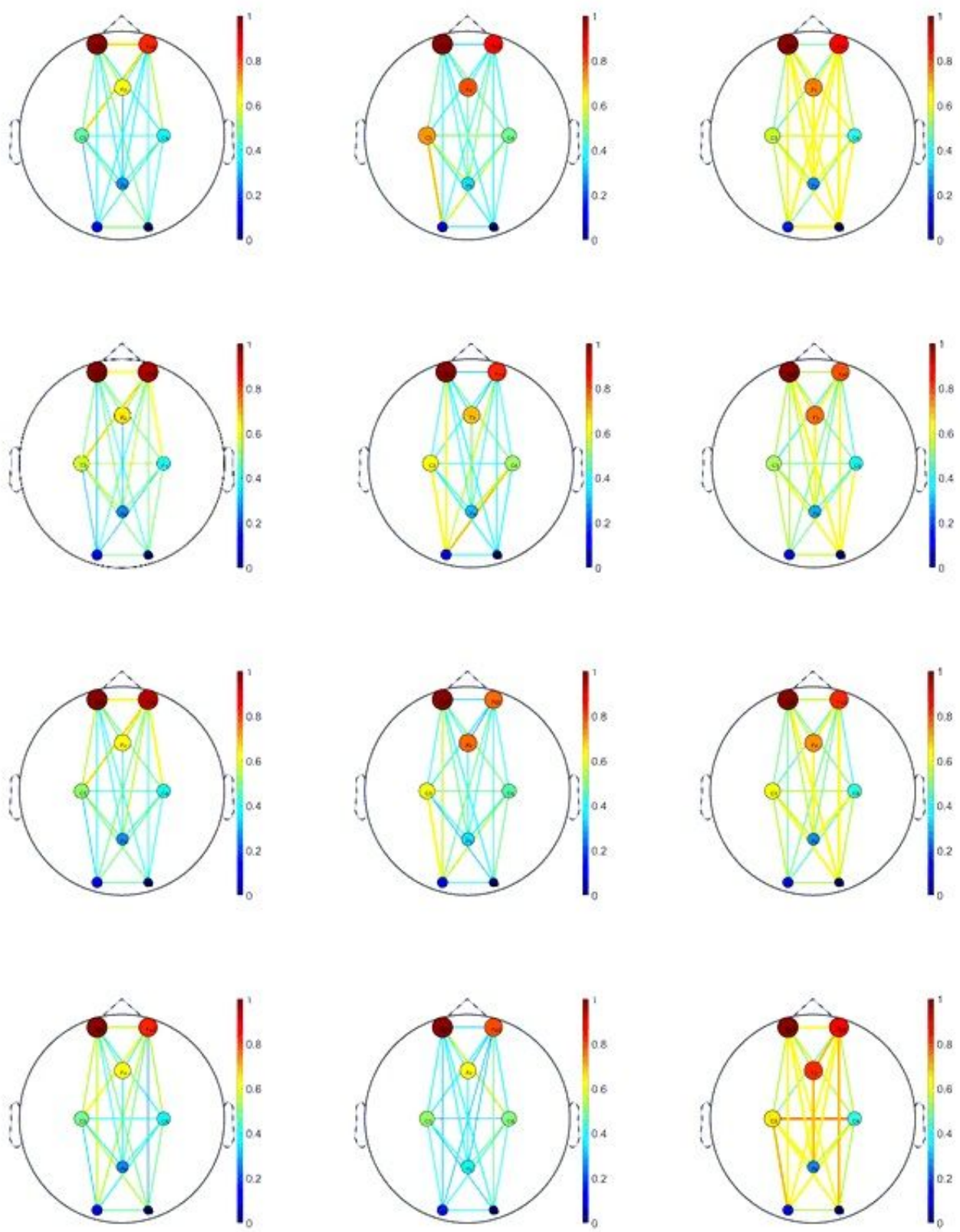

Figure 7

Task-related coherence in the three groups. ADHD, ADHD: attention deficit hyperactivity disorder; ADHD-H, ADHD with high cognitive proficiency; ADHD-L, ADHD with low cognitive proficiency; TD, typical development

\section{Supplementary Files}

This is a list of supplementary files associated with this preprint. Click to download.

- supplementtable131201.docx

- supplementtable131201.docx 